\title{
$N$-Methylpropargylamine-Conjugated Hydroxamic Acids as Dual Inhibitors of Monoamine Oxidase A and Histone Deacetylase for Glioma Treatment
}

Samir Mehndiratta, ${ }^{\dagger, l, \perp}$ Bin Qian, ${ }^{\dagger}$ Jian-Ying Chuang,,$^{\perp}$ Jing-Ping Liou, $,{ }^{*}, \|$ and Jean Chen Shih $^{*, \ldots, \dagger, \S, l}$

${ }^{\dagger}$ Department of Pharmacology and Pharmaceutical Sciences, School of Pharmacy, University of Southern California, Los Angeles, California 90089, United States.

$\star$ Department of Integrative Anatomical Sciences, Keck School of Medicine, University of Southern California, Los Angeles, California 90033, United States.

$\S$ USC- Taiwan Center for Translational Research, Los Angeles, California 90089, United States

"School of Pharmacy, College of Pharmacy, Taipei Medical University, Taipei 110, Taiwan.

${ }^{\perp}$ The Ph.D. Program for Neural Regenerative Medicine, College of Medical Science and Technology, Taipei Medical University, Taipei 110, Taiwan

\# TMU Research Center of Drug Discovery, Taipei Medical University, Taipei 110, Taiwan

1 *E-mail: jpl@tmu.edu.tw. Tel: 886-2-2736-1661 ext 6130

*E-mail: jcshih@usc.edu. Tel: (323) 442-1441 


\section{Content}

HPLC/UPLC purity data for compounds $8-15 \ldots \ldots \ldots \ldots \ldots \ldots \ldots \ldots . \ldots \ldots$

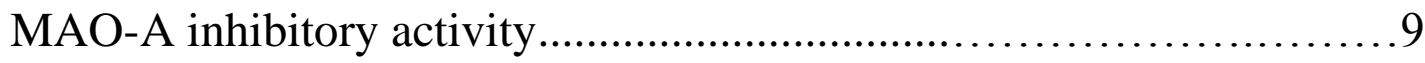

MAO B inhibition evaluation using Glo Assay..........................10

LSD1 Fluorescence coupling enzyme assay.........................11

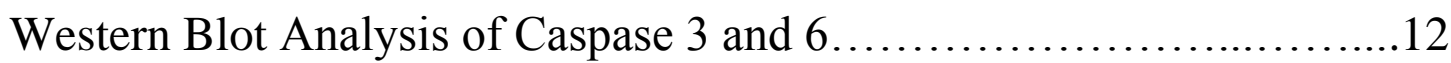

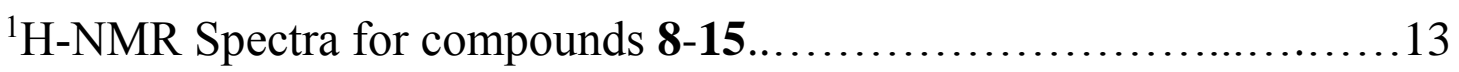

${ }^{13} \mathrm{C}-\mathrm{NMR}$ Spectra for compounds 8-15..........................21

HRMS Spectra for compounds 8 -15..............................29 
HPLC/UPLC purity data for compounds 8-15.

\section{HPLC/UPLC purity determination:}

The percentage purity of compounds was determined by an Agilent 1100 series HPLC system using C18 column or using Waters Acquity UPLC/BSM with PhotoDiode Array detector using Waters Acquity BEH-C18, $2.1 \mathrm{~mm}$ (ID) x $50 \mathrm{~mm}(\mathrm{~L}), 1.7 \mu \mathrm{m}$ particle size

Elution conditions for HPLC: Mobile phase A-Acetonitrile; Mobile phase B-Water containing $0.1 \%$ formic acid $+10 \mathrm{mM} \mathrm{NH}_{4} \mathrm{OAc}$. The flow-rate was $0.2 \mathrm{ml} / \mathrm{min}$ and the injection volume was $5 \mu$. The system operated at $25^{\circ} \mathrm{C}$. Peaks were detected at $210 \mathrm{~nm}$.

Elution conditions for UPLC: Mobile phase A-Acetonitrile; Mobile phase B-Water containing $0.1 \%$ formic acid $+2 \mathrm{mM} \mathrm{NH} \mathrm{H}_{4} \mathrm{OAc}$. The flow-rate was $0.6 \mathrm{ml} / \mathrm{min}$ and the injection volume was $5 \mu \mathrm{l}$. The system operated at $25^{\circ} \mathrm{C}$. Peaks were detected at $254 \mathrm{~nm}$.

Table S1. Elution condition HPLC

\begin{tabular}{|c|c|c|}
\hline Time (min) & Mobile Phase A (ratio) & Mobile Phase B (ratio) \\
\hline 0 & 10 & 90 \\
\hline 45 & 90 & 10 \\
\hline 50 & 10 & 90 \\
\hline 60 & 10 & 90 \\
\hline
\end{tabular}

Table S2. Elution condition UPLC

\begin{tabular}{|c|c|c|}
\hline $\begin{array}{c}\text { Time } \\
(\mathrm{min})\end{array}$ & \% A solvent & \% B solvent \\
\hline 0 & 90 & 10 \\
\hline 0.15 & 90 & 10 \\
\hline 4.15 & 10 & 90 \\
\hline 4.85 & 10 & 90 \\
\hline 5 & 90 & 10 \\
\hline 6.5 & 90 & 10 \\
\hline
\end{tabular}


Table S3. Purity data of compounds 8-15

\begin{tabular}{|c|c|c|}
\hline Compounds & Retention time (min) & $\%$ Purity UPLC \\
\hline \multirow{4}{*}{8} & 0.850 & 0.84 \\
\hline & 1.006 & 97.35 \\
\hline & 1.079 & 0.93 \\
\hline & 1.318 & 0.87 \\
\hline \multirow{4}{*}{9} & 0.851 & 0.41 \\
\hline & 1.005 & 98.79 \\
\hline & 1.079 & 0.44 \\
\hline & 1.317 & 0.37 \\
\hline \multirow{4}{*}{10} & 0.850 & 0.48 \\
\hline & 1.004 & 98.55 \\
\hline & 1.079 & 0.64 \\
\hline & 1.318 & 0.33 \\
\hline \multirow{7}{*}{14} & 1.173 & 0.55 \\
\hline & 1.301 & 97.12 \\
\hline & 1.393 & 0.76 \\
\hline & 1.634 & 0.36 \\
\hline & 1.661 & 0.50 \\
\hline & 1.998 & 0.14 \\
\hline & 2.027 & 0.57 \\
\hline \multirow{4}{*}{15} & 0.850 & 0.53 \\
\hline & 1.005 & 98.29 \\
\hline & 1.080 & 0.61 \\
\hline & 1.317 & 0.57 \\
\hline Compounds & Retention time (min) & $\%$ Purity HPLC \\
\hline \multirow{7}{*}{11} & 15.97 & 1.356 \\
\hline & 16.89 & 95.82 \\
\hline & 17.69 & 1.049 \\
\hline & 20.21 & 0.631 \\
\hline & 21.34 & 0.403 \\
\hline & 22.38 & 0.351 \\
\hline & 22.75 & 0.386 \\
\hline \multirow{8}{*}{12} & 13.39 & 0.683 \\
\hline & 15.05 & 95.80 \\
\hline & 16.00 & 0.557 \\
\hline & 18.31 & 1.229 \\
\hline & 18.69 & 0.276 \\
\hline & 19.77 & 0.169 \\
\hline & 20.63 & 0.169 \\
\hline & 22.73 & 0.295 \\
\hline \multirow{6}{*}{13} & 15.08 & 0.336 \\
\hline & 16.57 & 98.37 \\
\hline & 19.53 & 0.395 \\
\hline & 19.85 & 0.193 \\
\hline & 20.37 & 0.316 \\
\hline & 22.65 & 0.381 \\
\hline
\end{tabular}


Purity Compound $\mathbf{8}$

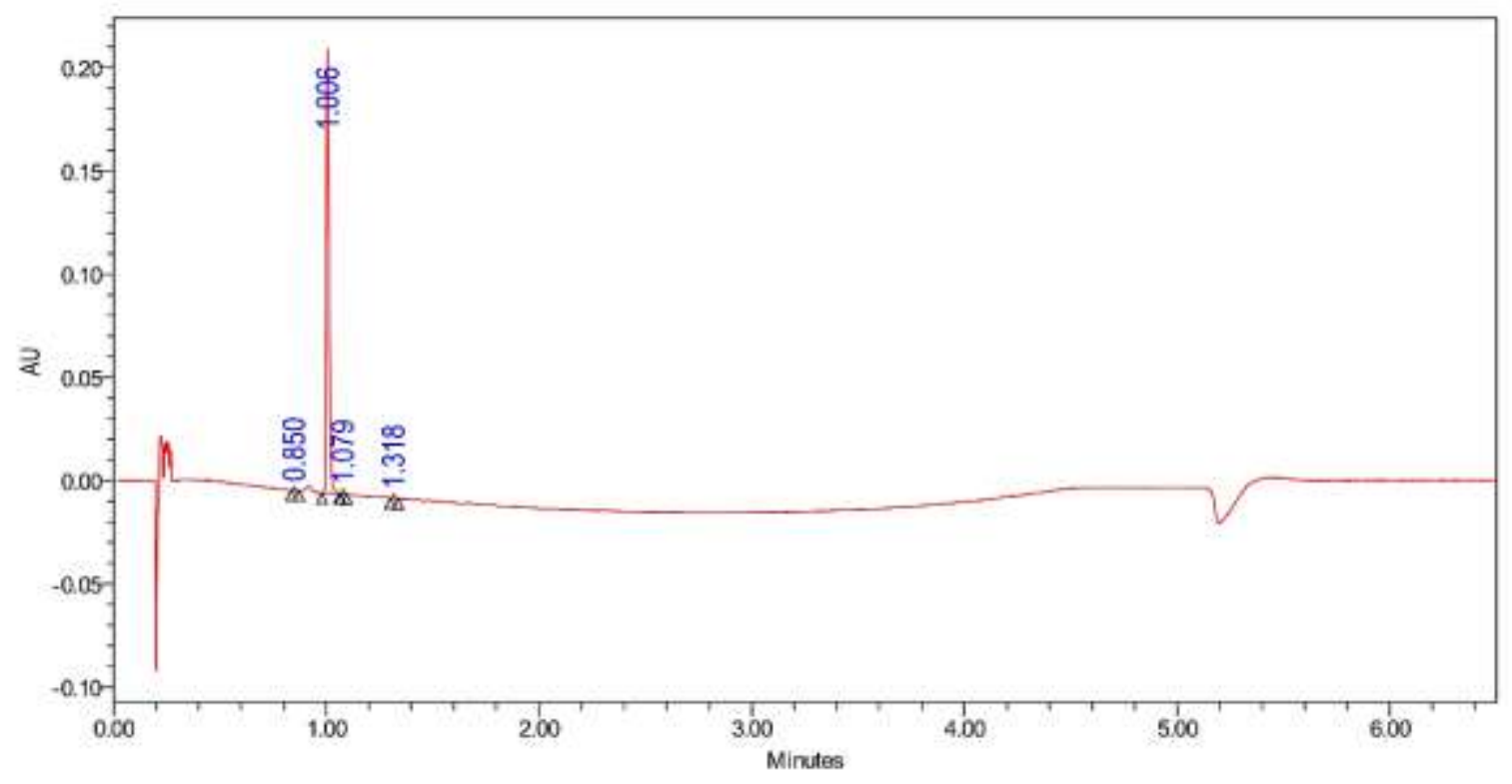

\begin{tabular}{|l|c|r|r|r|}
\hline & RT & Height & Area & $\%$ Area \\
\hline 1 & 0.850 & 1892 & 1667 & 0.84 \\
\hline 2 & 1.006 & 215067 & 192664 & 97.35 \\
\hline 3 & 1.079 & 2493 & 1845 & 0.93 \\
\hline 4 & 1.318 & 2150 & 1728 & 0.87 \\
\hline
\end{tabular}

Purity Compound 9

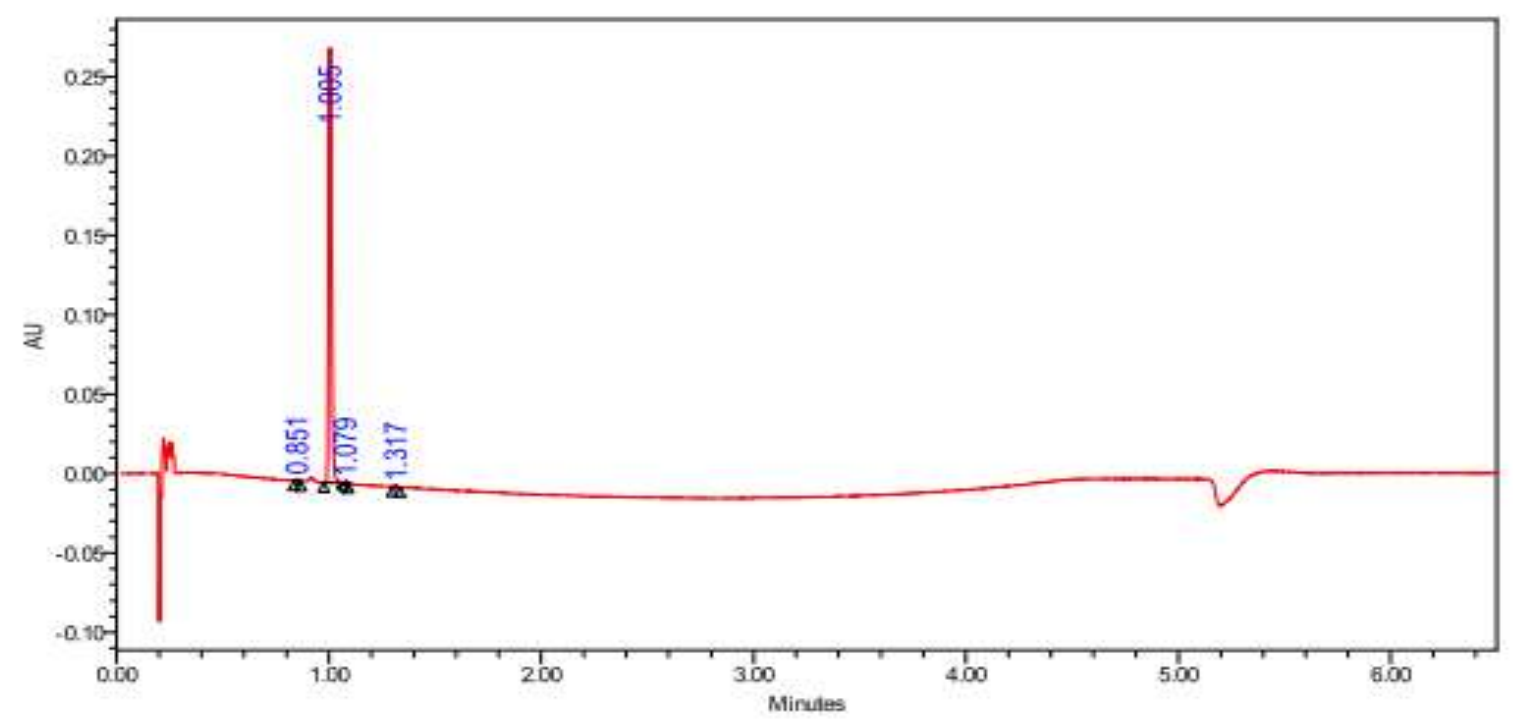

\begin{tabular}{|r|c|r|r|r|}
\hline & RT & Height & Area & \% Area \\
\hline 1 & 0.851 & 1344 & 1050 & 0.41 \\
\hline 2 & 1.005 & 273830 & 255862 & 98.79 \\
\hline 3 & 1.079 & 1565 & 1138 & 0.44 \\
\hline 4 & 1.317 & 1108 & 954 & 0.37 \\
\hline
\end{tabular}


Purity Compound $\mathbf{1 0}$

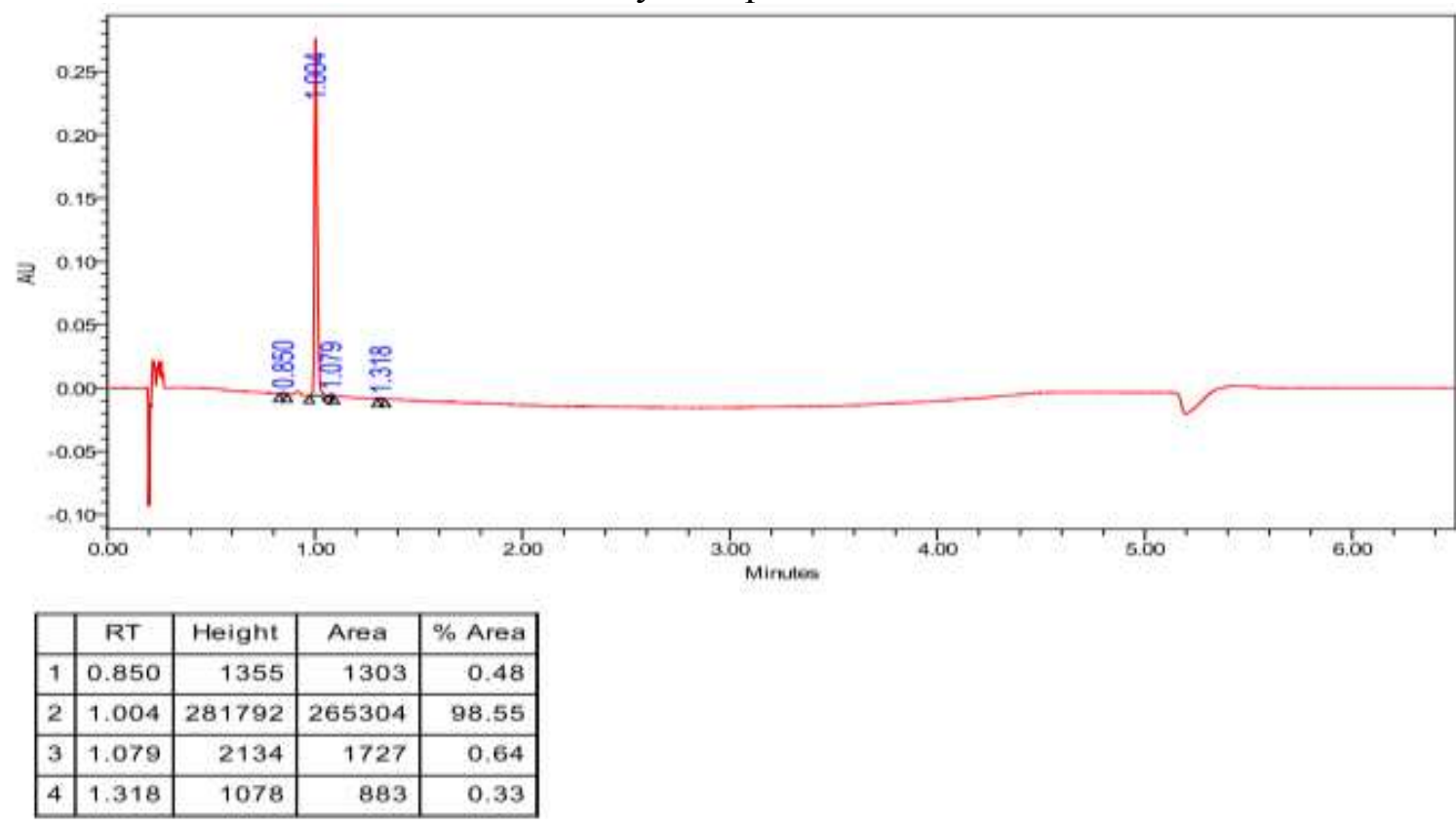

Purity Compound 11

Chrom Type: Fixed WL Chromatogram, 254 nm

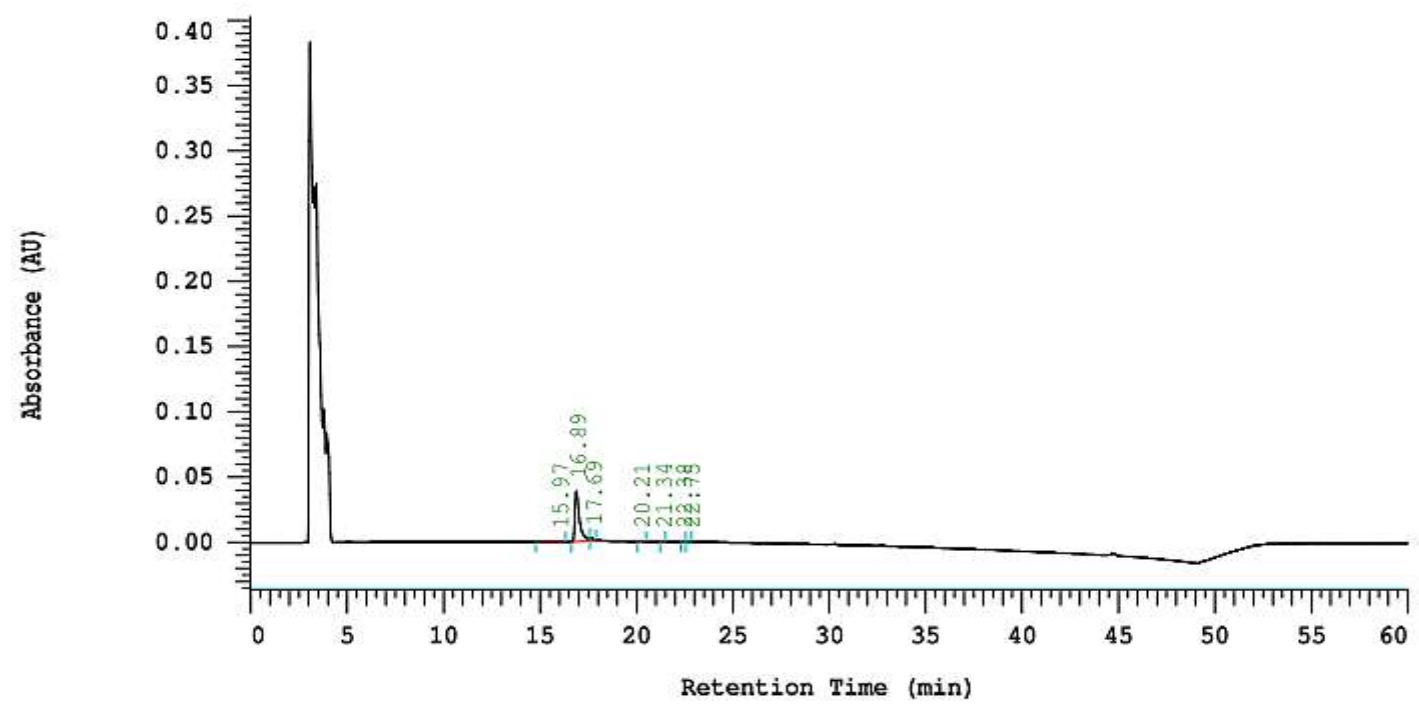

Processing Method: Purity_60min

Column Type: Column

Method Developer: Bob

Method Description:

Peak Quantitation: AREA

Calculation Method: AREA

No.

RT

Area

Height

Conc 1

\begin{tabular}{rrrrr}
1 & 15.97 & 4649 & 216 & 1.356 \\
2 & 16.89 & 328514 & 19318 & 95.825 \\
3 & 17.69 & 3595 & 436 & 1.049 \\
4 & 20.21 & 2161 & 245 & 0.631 \\
5 & 21.34 & 1381 & 167 & 0.403 \\
6 & 22.38 & 1204 & 171 & 0.351 \\
7 & 22.75 & 1321 & 150 & 0.386 \\
\hline & & 342825 & 20703 & 100.000 \\
\hline
\end{tabular}




\section{Purity Compound 12}

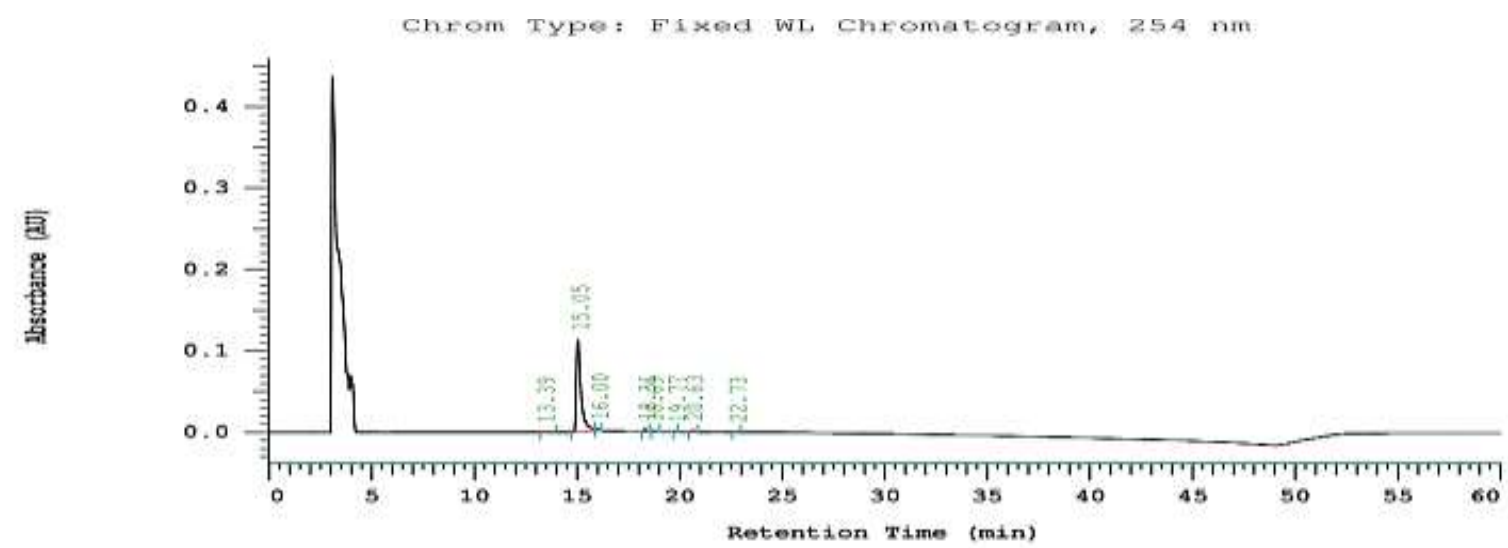

Proceseing Method: Purity-60min Column TYPe: Column Method Descipiption:

Method Developax: Bob

Deak Quantitation: AREA

Calculattor Mothod: AREA

\begin{tabular}{|c|c|c|c|c|}
\hline No, & $\mathrm{R} T$ & Axea & Height & cone 1 \\
\hline 1 & 13.39 & 6208 & 446 & 0.683 \\
\hline 2 & 15.05 & 871090 & 56322 & 95.801 \\
\hline 3 & 16.00 & 5063 & 585 & 0.557 \\
\hline 4 & 18.31 & 11175 & 1339 & 1.22 .9 \\
\hline 5 & 18.69 & 2513 & 177 & 0.276 \\
\hline 6 & 19.77 & 1541 & 207 & 0.169 \\
\hline 7 & 20.63 & 8991 & 985 & 0.989 \\
\hline \multirow[t]{2}{*}{8} & 22.73 & 2685 & 255 & 0.295 \\
\hline & & 909266 & 60316 & 100.000 \\
\hline
\end{tabular}

\section{Purity Compound 13}

chrom Type: Fixed WL Chromatogram, 254 nm

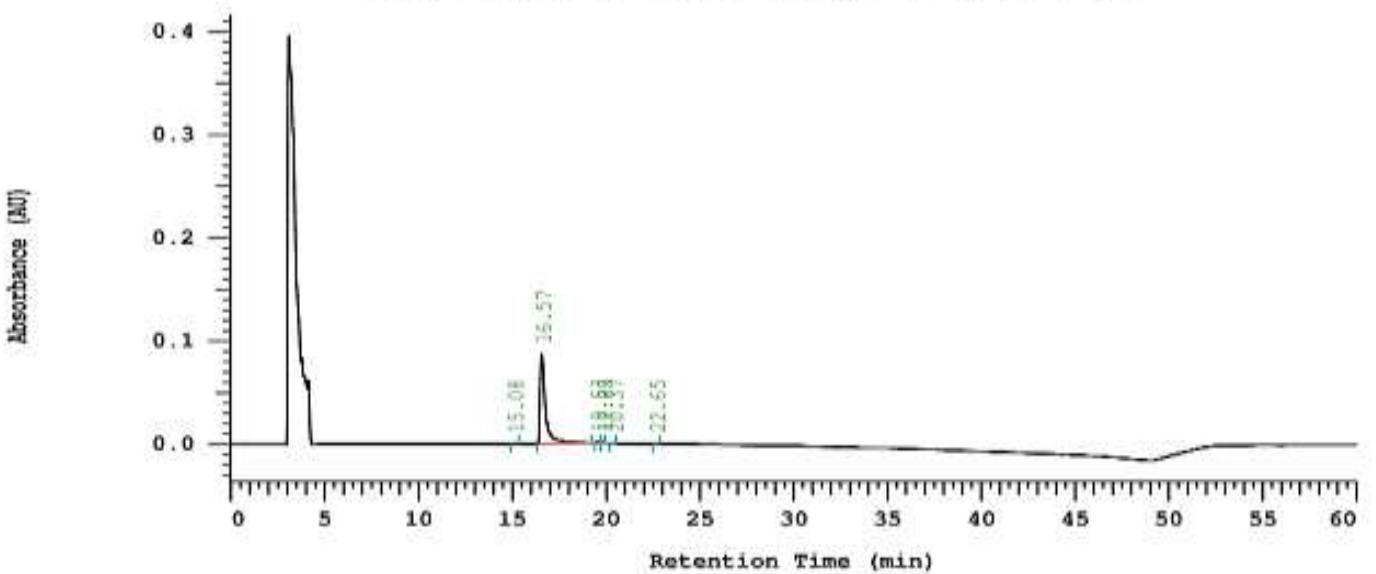

Processing Method: Purity_6omin

Column Type: Column

Method Developer; Bob

Method Description:

Peak Quantitation: AREA

Calculation Method: AREA?

\begin{tabular}{rrrrr} 
No, RT & \multicolumn{1}{c}{ Area } & Height & Cone 1 \\
\hline 1 & 15.08 & 2808 & 225 & 0.336 \\
2 & 16.57 & 821919 & 43442 & 98.379 \\
3 & 19.53 & 3298 & 921 & 0.395 \\
4 & 19.85 & 1610 & 209 & 0.193 \\
5 & 20.37 & 2644 & 289 & 0.316 \\
6 & 22.65 & 3180 & 330 & 0.381 \\
\hline & & 835459 & 44916 & 100.000 \\
\hline
\end{tabular}


Purity Compound 14

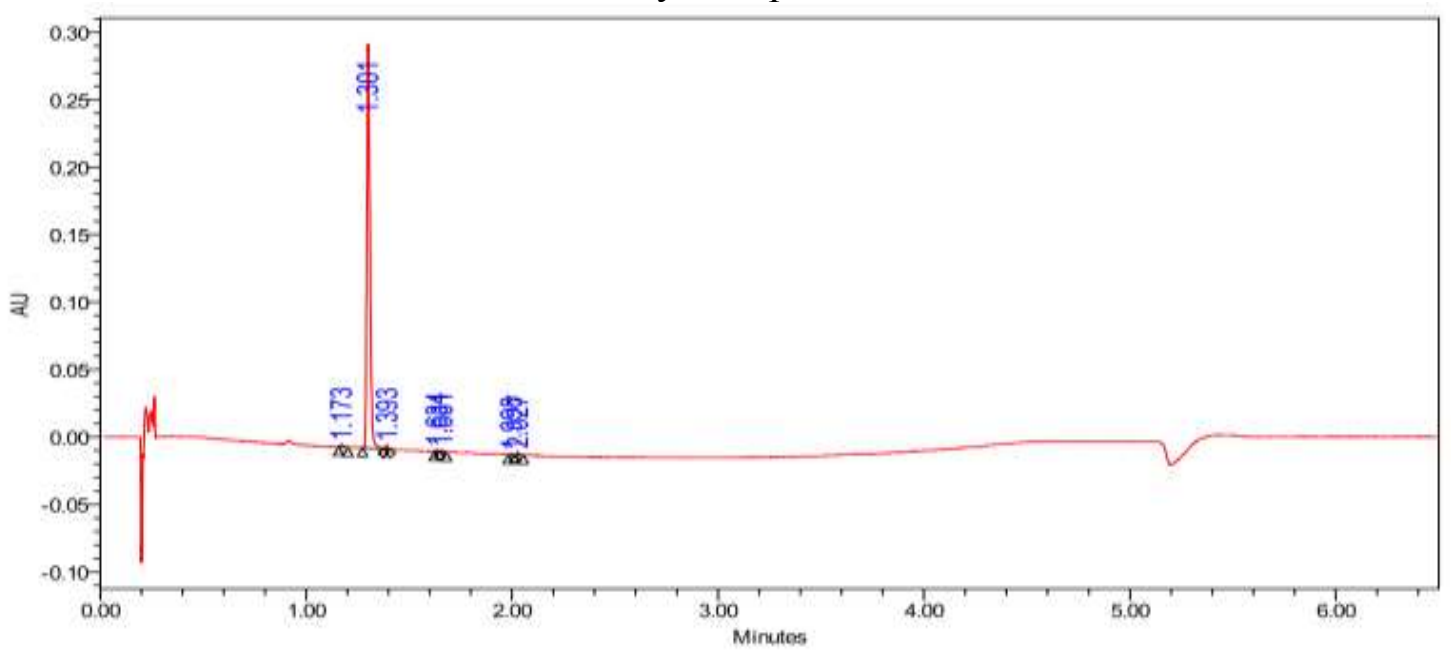

\begin{tabular}{|r|c|r|r|r|}
\hline & RT & Height & Area & \% Area \\
\hline 1 & 1.173 & 2069 & 1782 & 0.55 \\
\hline 2 & 1.301 & 300048 & 315341 & 97.12 \\
\hline 3 & 1.393 & 2833 & 2462 & 0.76 \\
\hline 4 & 1.634 & 1361 & 1181 & 0.36 \\
\hline 5 & 1.661 & 1563 & 1635 & 0.50 \\
\hline 6 & 1.998 & 613 & 452 & 0.14 \\
\hline
\end{tabular}

\begin{tabular}{|l|c|r|r|r|}
\hline & RT & Height & Area & $\%$ Area \\
\hline 7 & 2.027 & 1970 & 1846 & 0.57 \\
\hline
\end{tabular}

Purity Compound 15

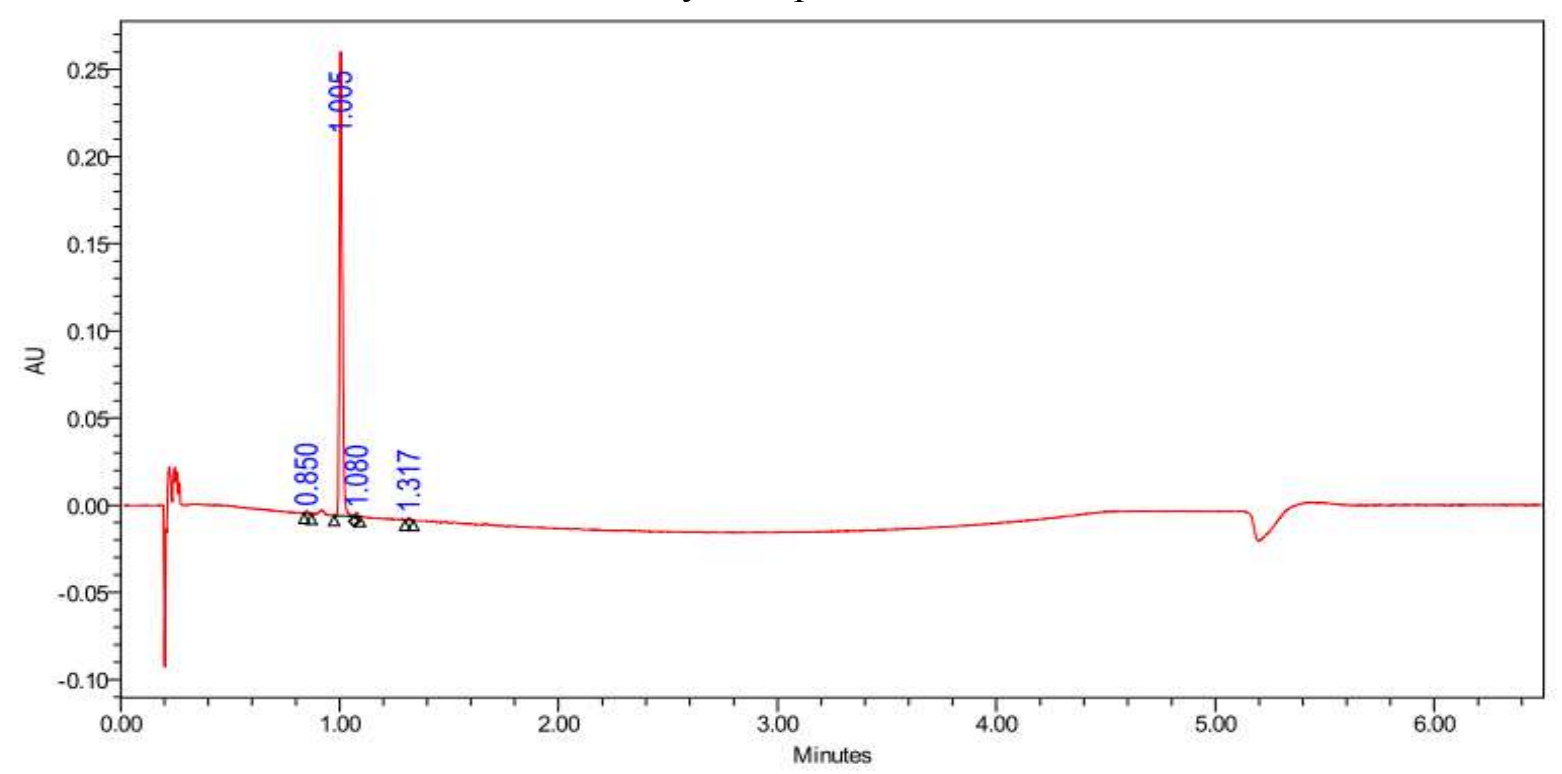

\begin{tabular}{|l|c|r|r|r|}
\hline & RT & Height & Area & $\%$ Area \\
\hline 1 & 0.850 & 1602 & 1345 & 0.53 \\
\hline 2 & 1.005 & 266108 & 248187 & 98.29 \\
\hline 3 & 1.080 & 2106 & 1535 & 0.61 \\
\hline 4 & 1.317 & 1578 & 1441 & 0.57 \\
\hline
\end{tabular}


MAO-A inhibitory activity

Table S4. Inhibition of MAO-A by MAO-A and HDAC dual inhibitors

\begin{tabular}{|c|c|}
\hline Compound & MAO-A inhibitory $\mathrm{IC}_{50}$ GL26 \\
\hline $\mathbf{8}$ & $0.03 \pm 0.16 \mu \mathrm{M}$ \\
\hline $\mathbf{9}$ & $23.30 \pm 0.09 \mu \mathrm{M}$ \\
\hline $\mathbf{1 0}$ & $3.25 \pm 0.12 \mu \mathrm{M}$ \\
\hline $\mathbf{1 1}$ & $0.43 \pm 0.08 \mu \mathrm{M}$ \\
\hline $\mathbf{1 2}$ & $0.02 \pm 0.02 \mu \mathrm{M}$ \\
\hline $\mathbf{1 3}$ & $0.002 \mathrm{nM}$ \\
\hline $\mathbf{1 4}$ & $4.00 \mathrm{nM}$ \\
\hline $\mathbf{1 5}$ & $0.40 \mathrm{nM}$ \\
\hline SAHA (1) & $(-)$ \\
\hline Clorgyline (5) & $0.10 \mathrm{nM}$ \\
\hline
\end{tabular}

${ }^{36}$ : 2015, WO 2015/120206 A1; (-): No Activity 
MAO B inhibition evaluation using Glo Assay

A

\begin{tabular}{|c|c|c|}
\hline Compound & $\begin{array}{c}\text { \% Inhibition of } \\
\text { MAO B (brain) } \\
\text { at } 10 \mu \mathrm{M}\end{array}$ & $\begin{array}{c}\text { \% Inhibition of } \\
\text { MAO B (brain) } \\
\text { at 1 } \mu \mathrm{M}\end{array}$ \\
\hline 8 & 65.7 & 11.6 \\
\hline 9 & 94.3 & 22.3 \\
\hline 10 & 90.3 & 22.2 \\
\hline 11 & 13.6 & 9.8 \\
\hline 12 & 94.5 & 22.7 \\
\hline 13 & 96.4 & 22.9 \\
\hline 14 & 87.1 & 22.2 \\
\hline 15 & 71.8 & 25.8 \\
\hline SAHA $^{24}$ & - & - \\
\hline Clorgyline $^{25}$ & 88.6 & 55.1 \\
\hline
\end{tabular}

B

MAO B-WT brain

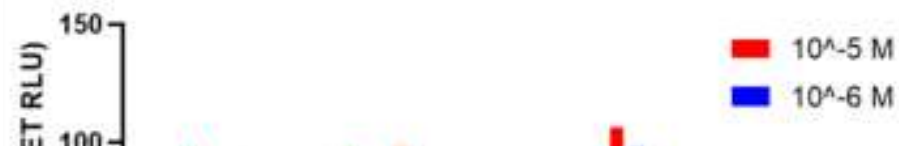

Figure S1. MAO B inhibition ability of clorgyline and SAHA based dual inhibitors of MAO A and HDAC. Concentration $10^{-5} \mathrm{M}=10 \mu \mathrm{M}$ and $10^{-6} \mathrm{M}=1 \mu \mathrm{M}$ 
LSD1 Fluorescence coupling enzyme assay

Table S5: Percentage inhibition of LSD1 by MAO-A and HDAC dual inhibitors $\mathbf{8}, \mathbf{1 3}, \mathbf{1 4}$, and 15.

\begin{tabular}{|c|c|c|c|c|}
\hline Concentration & $\mathbf{8} \%$ Inhib. & $\mathbf{1 3} \%$ Inhib. & $\mathbf{1 4} \%$ Inhib. & $\mathbf{1 5} \%$ Inhib. \\
\hline $10 \mu \mathrm{M}$ & 94.76 & 88.08 & 44.90 & 30.54 \\
\hline $3.33 \mu \mathrm{M}$ & 107.09 & 92.41 & 78.89 & 59.97 \\
\hline $1.11 \mu \mathrm{M}$ & 102.57 & 92.53 & 83.66 & 73.35 \\
\hline $0.37 \mu \mathrm{M}$ & 108.37 & 100.78 & 87.94 & 78.65 \\
\hline $0.12 \mu \mathrm{M}$ & 102.07 & 99.10 & 89.61 & 84.55 \\
\hline $0.04 \mu \mathrm{M}$ & 108.86 & 102.15 & 94.80 & 87.72 \\
\hline $0.01 \mu \mathrm{M}$ & 111.26 & 97.84 & 95.58 & 86.86 \\
\hline $0.004 \mu \mathrm{M}$ & 104.22 & 104.99 & 94.76 & 87.55 \\
\hline $0.001 \mu \mathrm{M}$ & 100.78 & 91.94 & 94.65 & 90.00 \\
\hline $0.0005 \mu \mathrm{M}$ & 98.75 & 98.24 & 95.83 & 87.30 \\
\hline $\mathrm{DMSO}$ & 92.48 & 103.17 & 96.35 & 92.54 \\
\hline
\end{tabular}

Compound IC50 for LSD1

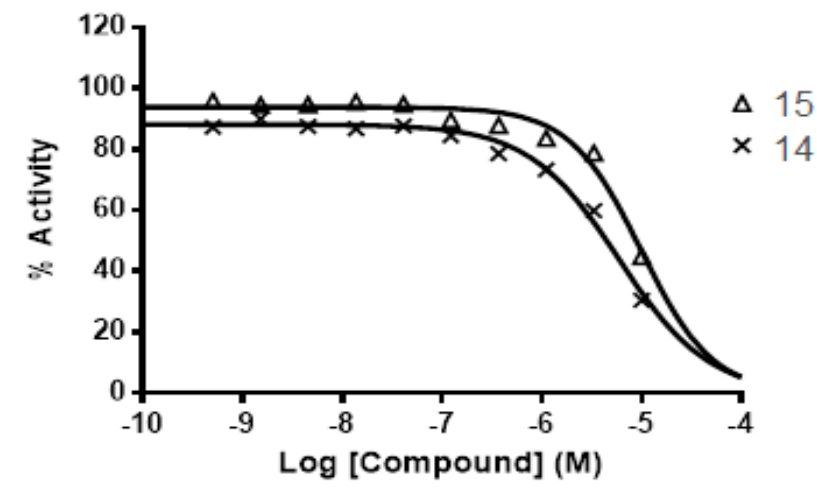

\begin{tabular}{l|ll} 
& \multicolumn{1}{|c}{15} & \multicolumn{1}{c}{14} \\
\hline HillSlope & -1.214 & -0.9650 \\
EC50 & $9.912 \mathrm{e}-006$ & $5.914 \mathrm{e}-006$
\end{tabular}

Figure S2. LSD1 inhibition ability of Clorgyline and SAHA based dual inhibitors of MAO A and HDAC 14 and 15. Concentration $1 \mathrm{e}-006=1 \times 10^{-6} \mathrm{M}=1 \mu \mathrm{M} ; 14 \mathrm{EC}_{50}: 5.9 \mu \mathrm{M} ; 15 \mathrm{EC}_{50}=9.9 \mu \mathrm{M}$. 
Western Blot Analysis of Caspase 3 and 6

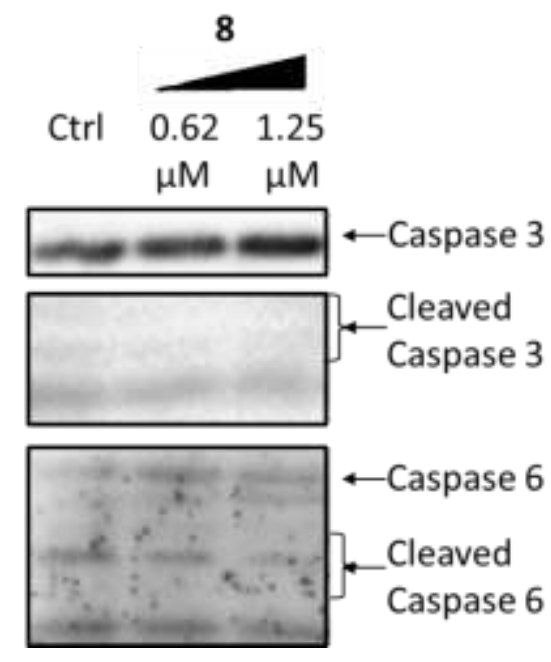

Figure S3. Western blot analysis of Caspase 3 and 6 activation after treatment with compound 8 . 


\section{${ }^{1} \mathrm{H}$ Spectrum for compound 8}
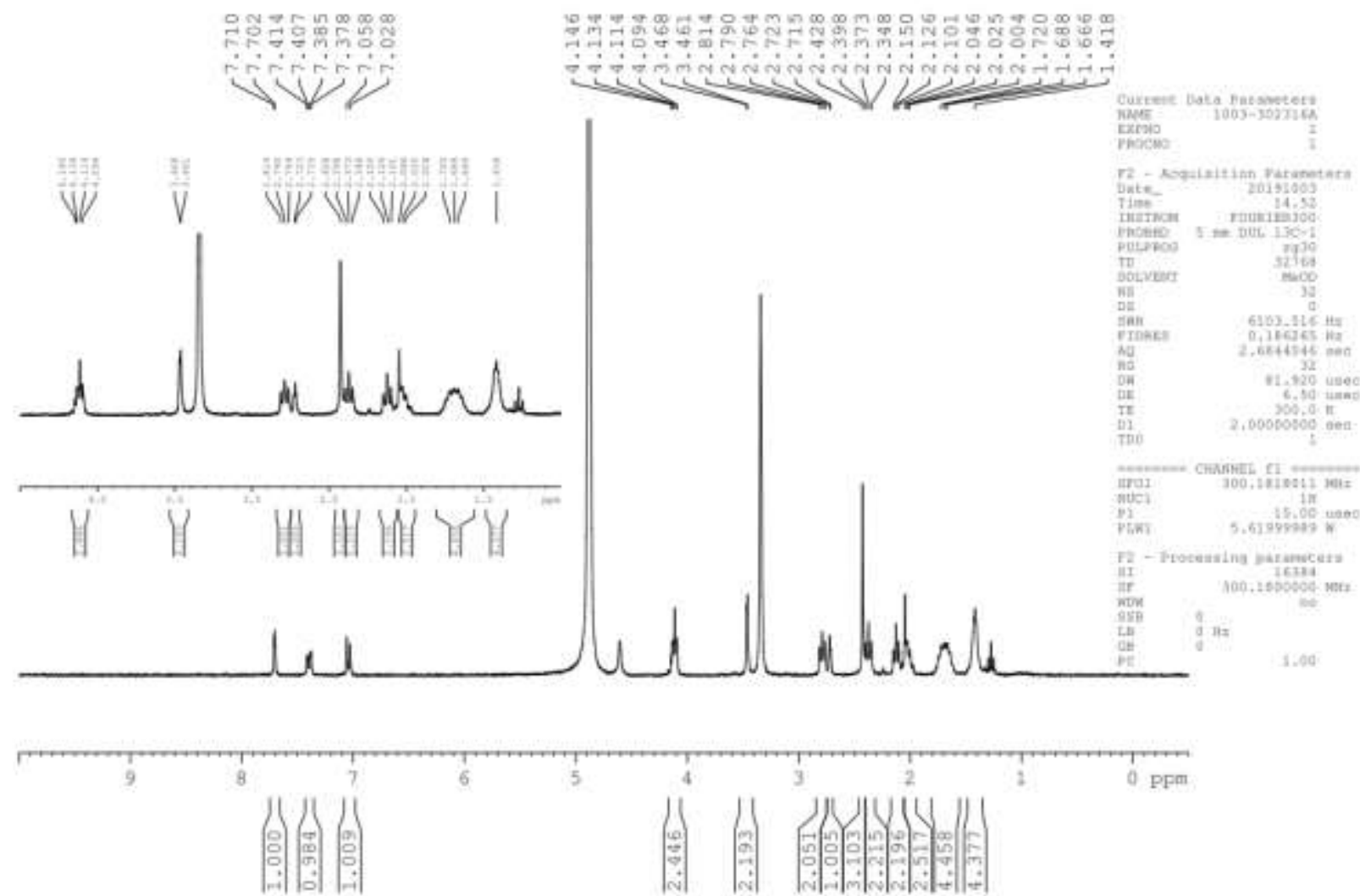
${ }^{1} \mathrm{H}$ Spectrum for compound 9

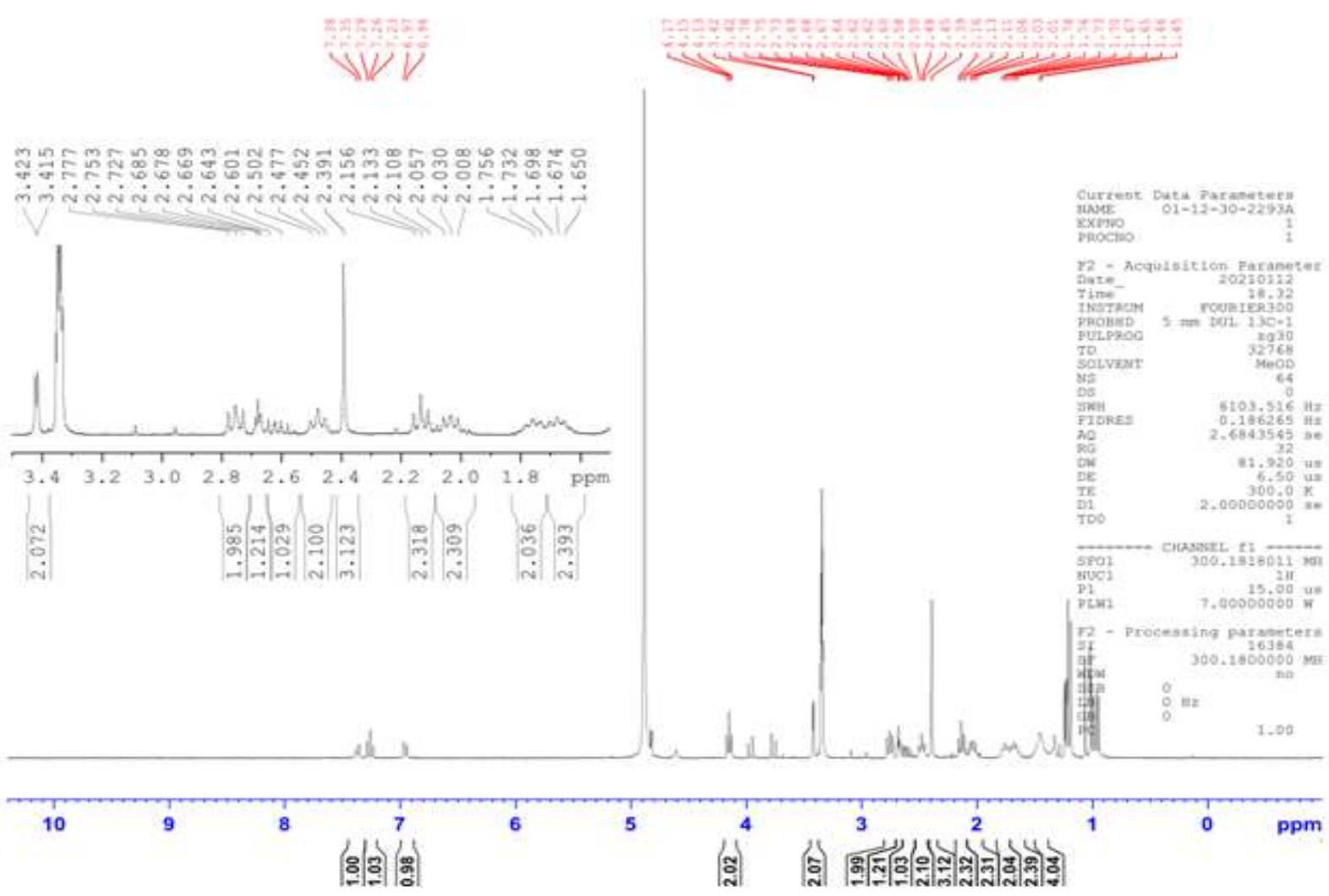




\section{${ }^{1} \mathrm{H}$ Spectrum for compound 10}

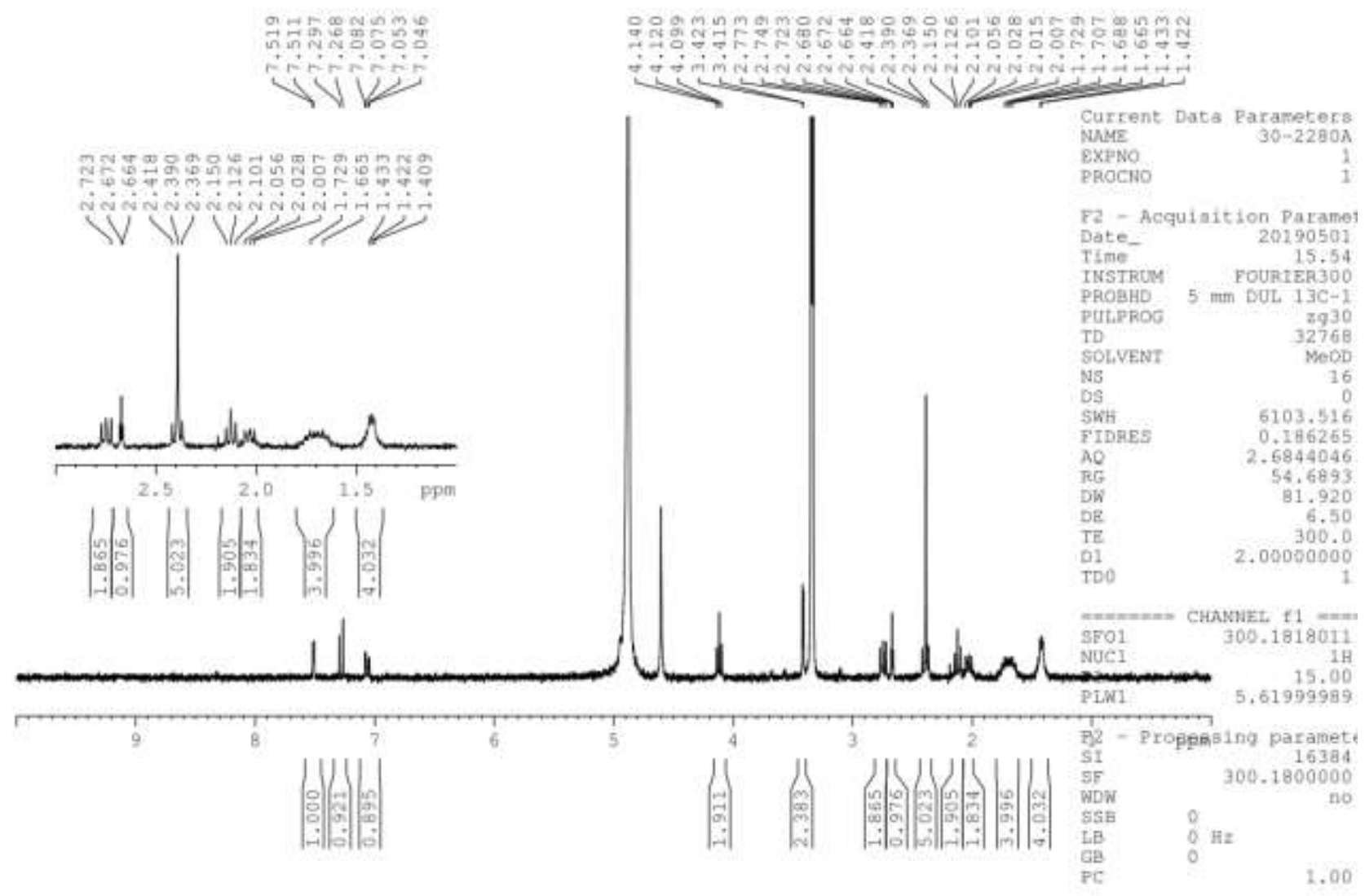


${ }^{1} \mathrm{H}$ Spectrum for compound 11

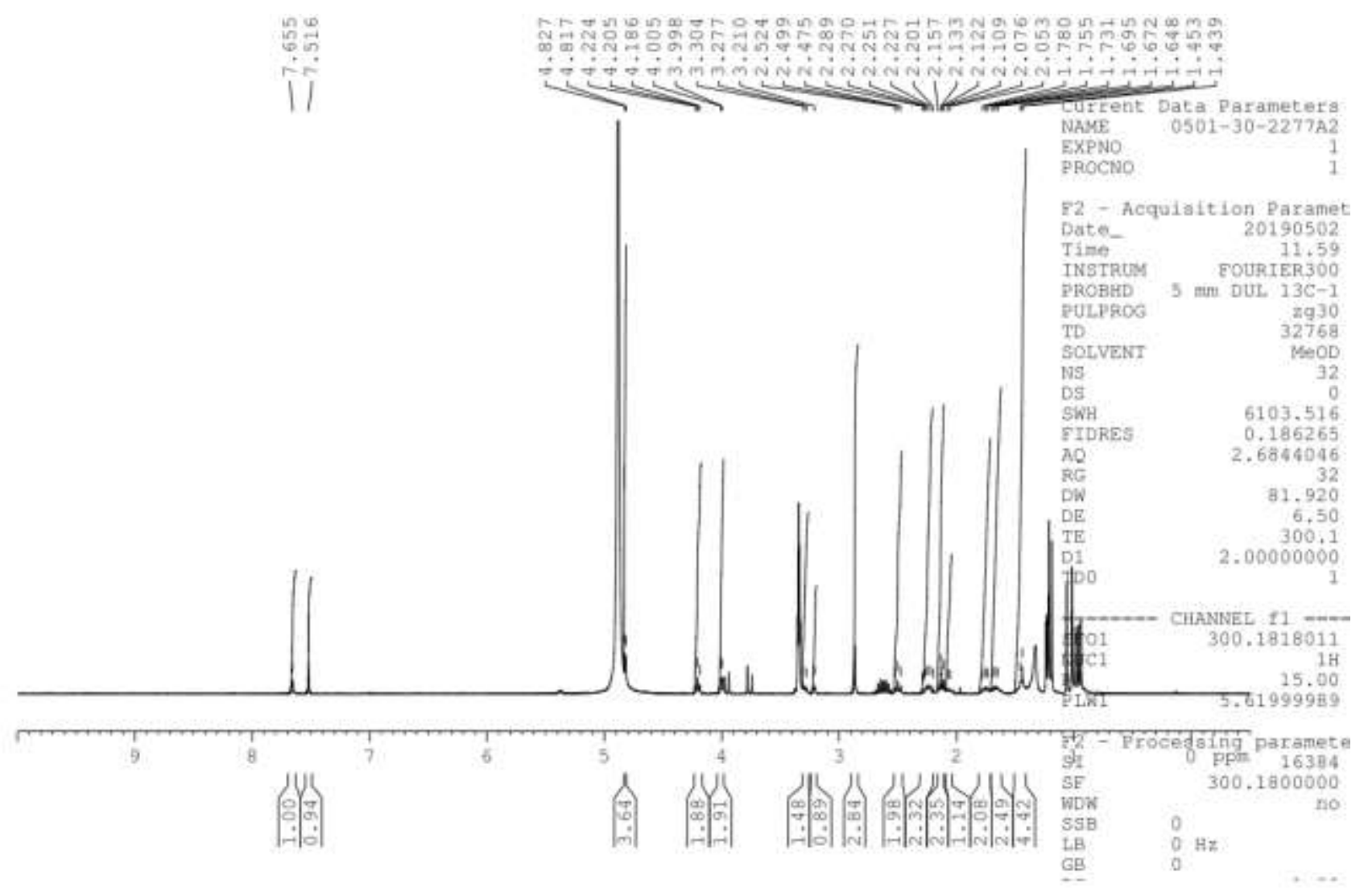




\section{${ }^{1} \mathrm{H}$ Spectrum for compound 12}

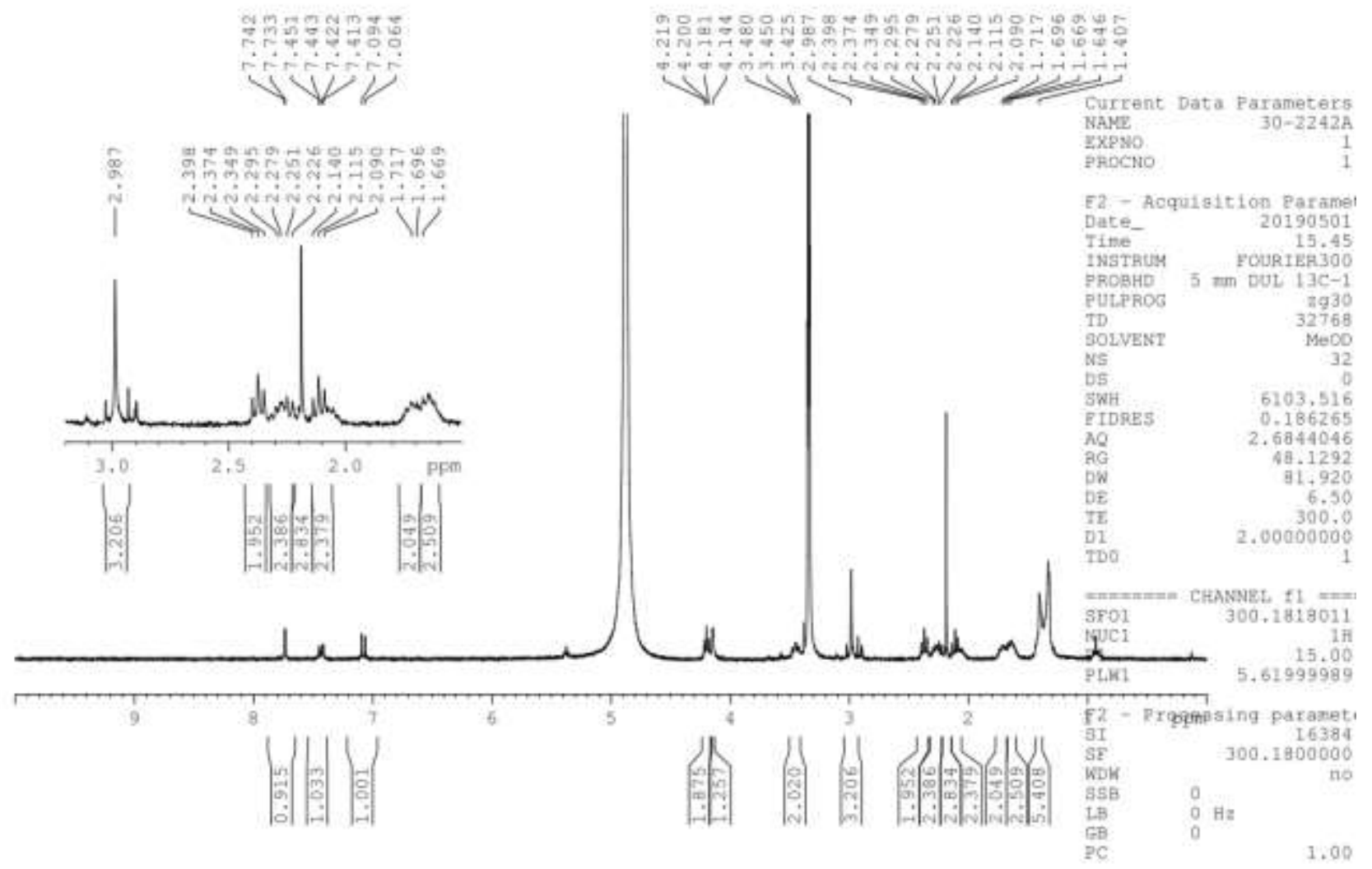




\section{SI-18}

\section{${ }^{1} \mathrm{H}$ Spectrum for compound 13}

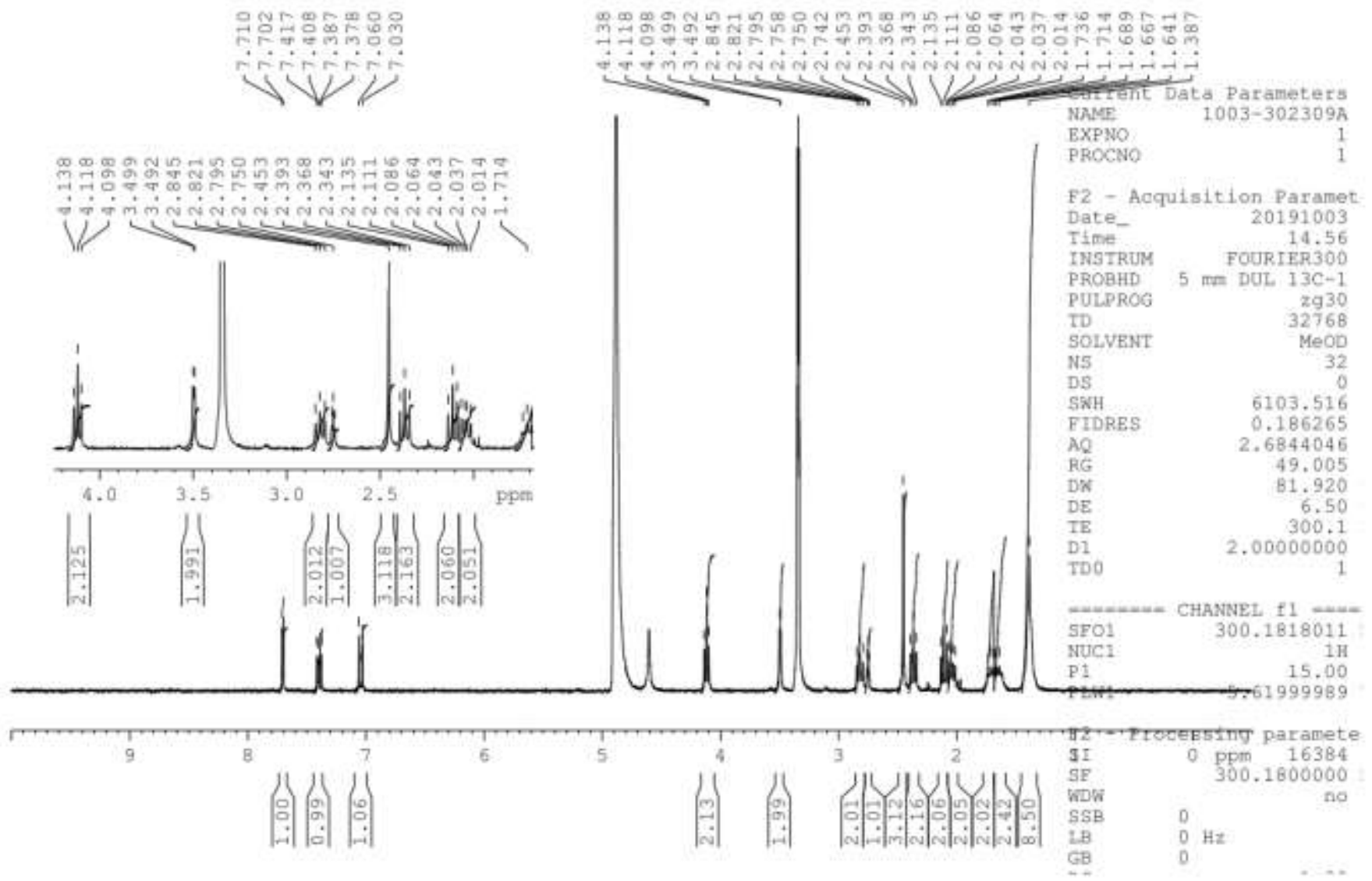




\section{${ }^{1} \mathrm{H}$ Spectrum for compound 14}

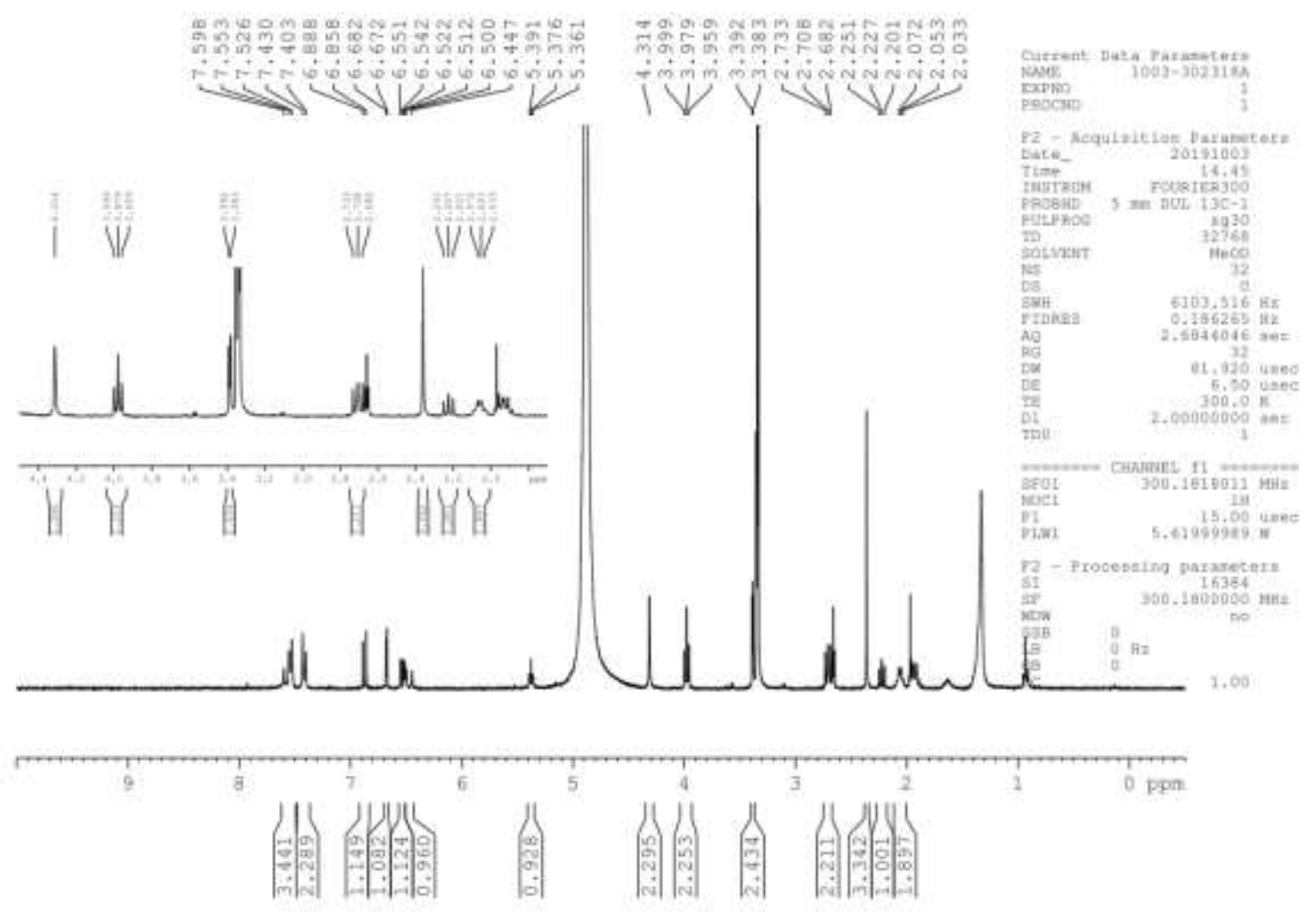


${ }^{1} \mathrm{H}$ Spectrum for compound 15

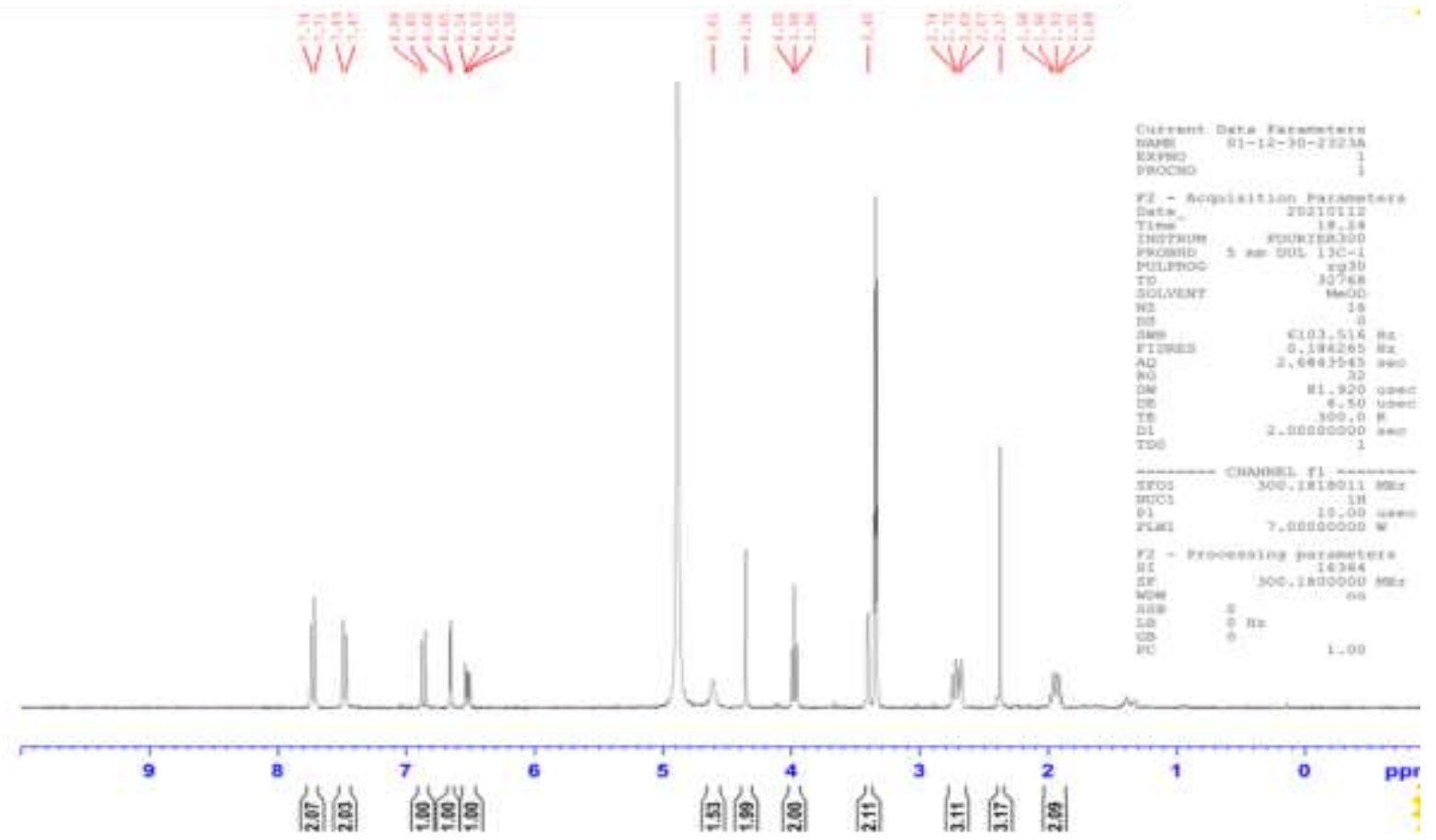


${ }^{13} \mathrm{C}$ Spectrum for compound 8

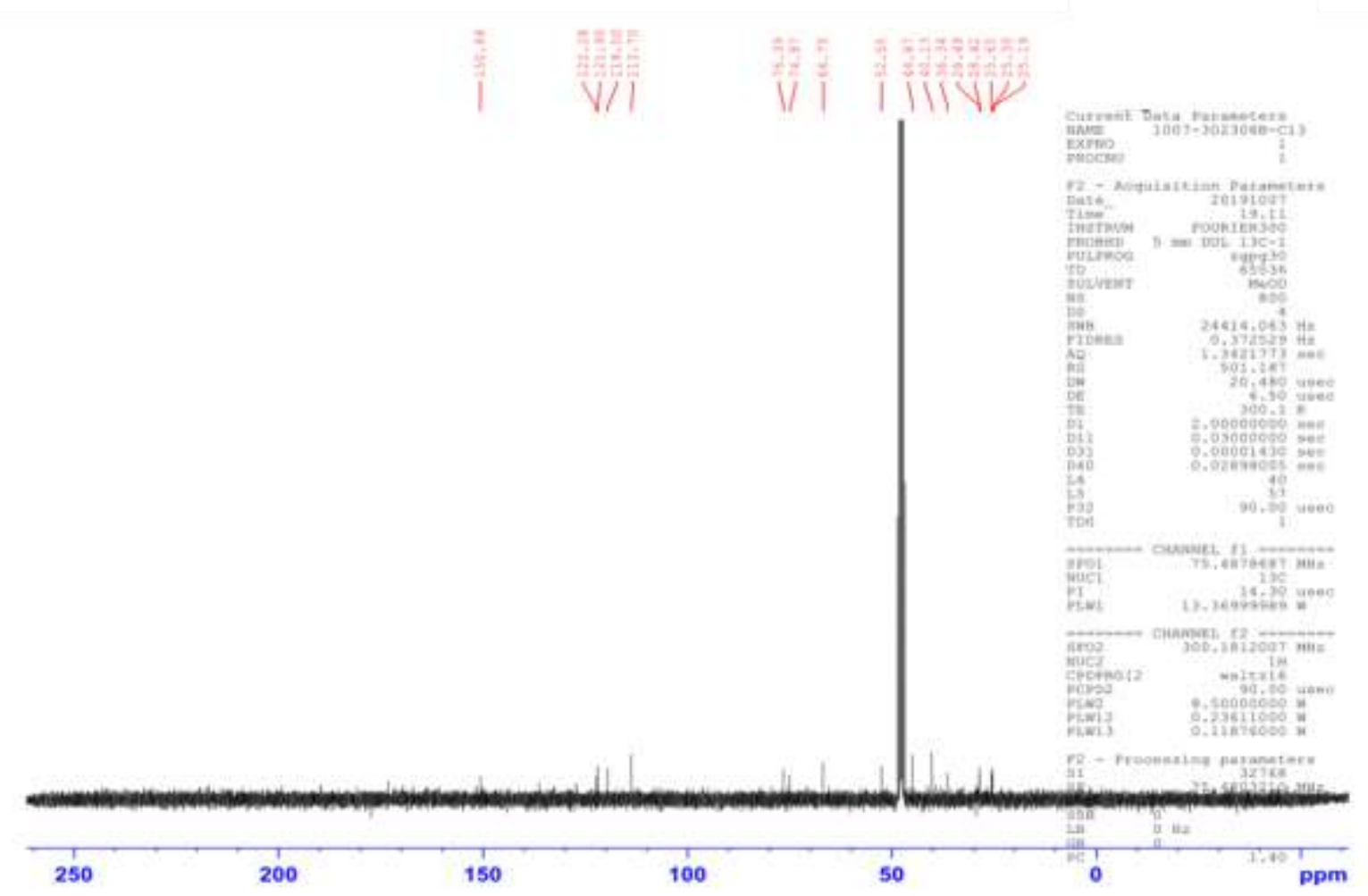


${ }^{13} \mathrm{C}$ Spectrum for compound 9

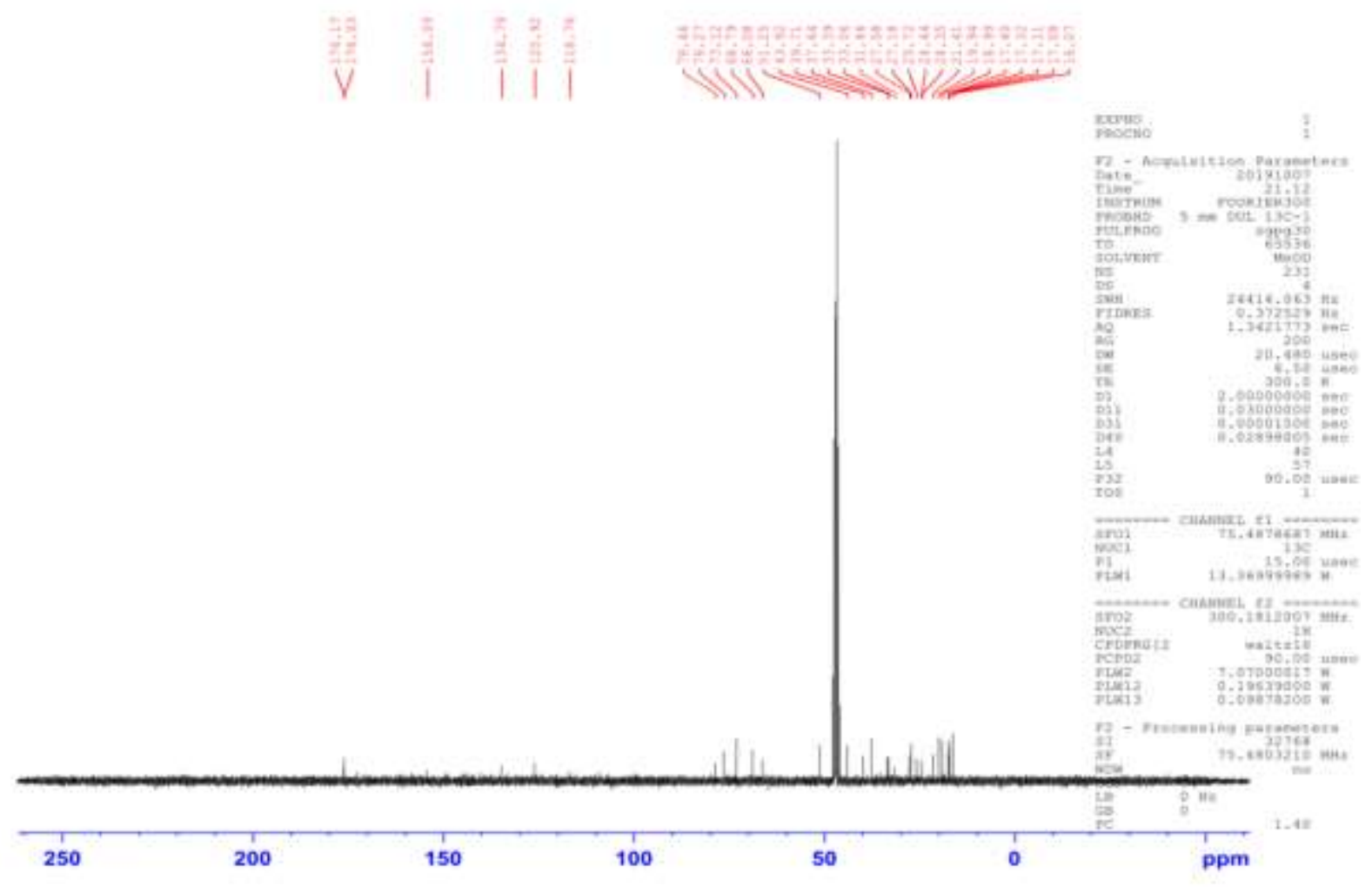


${ }^{13} \mathrm{C}$ Spectrum for compound 10

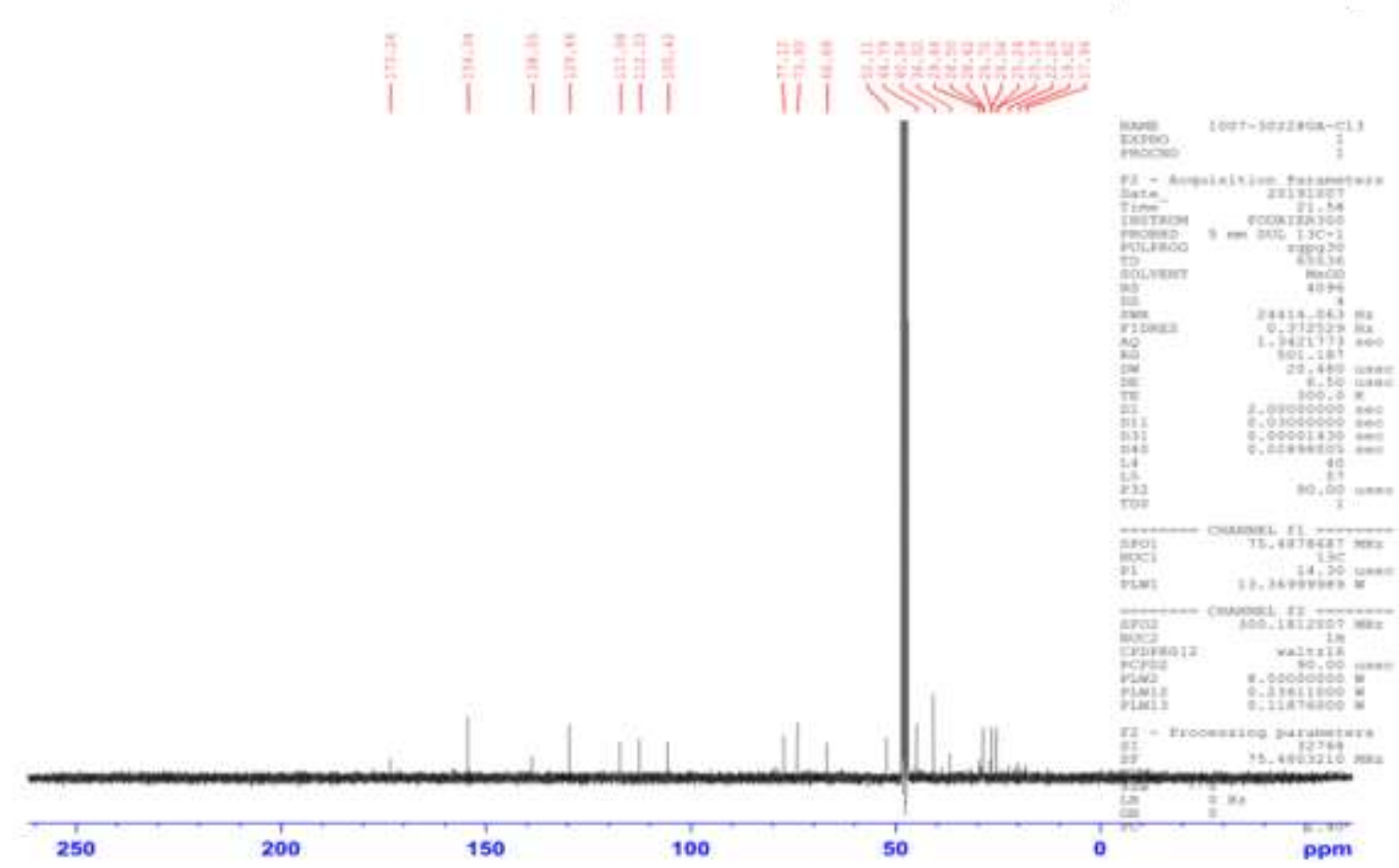


${ }^{13} \mathrm{C}$ Spectrum for compound 11

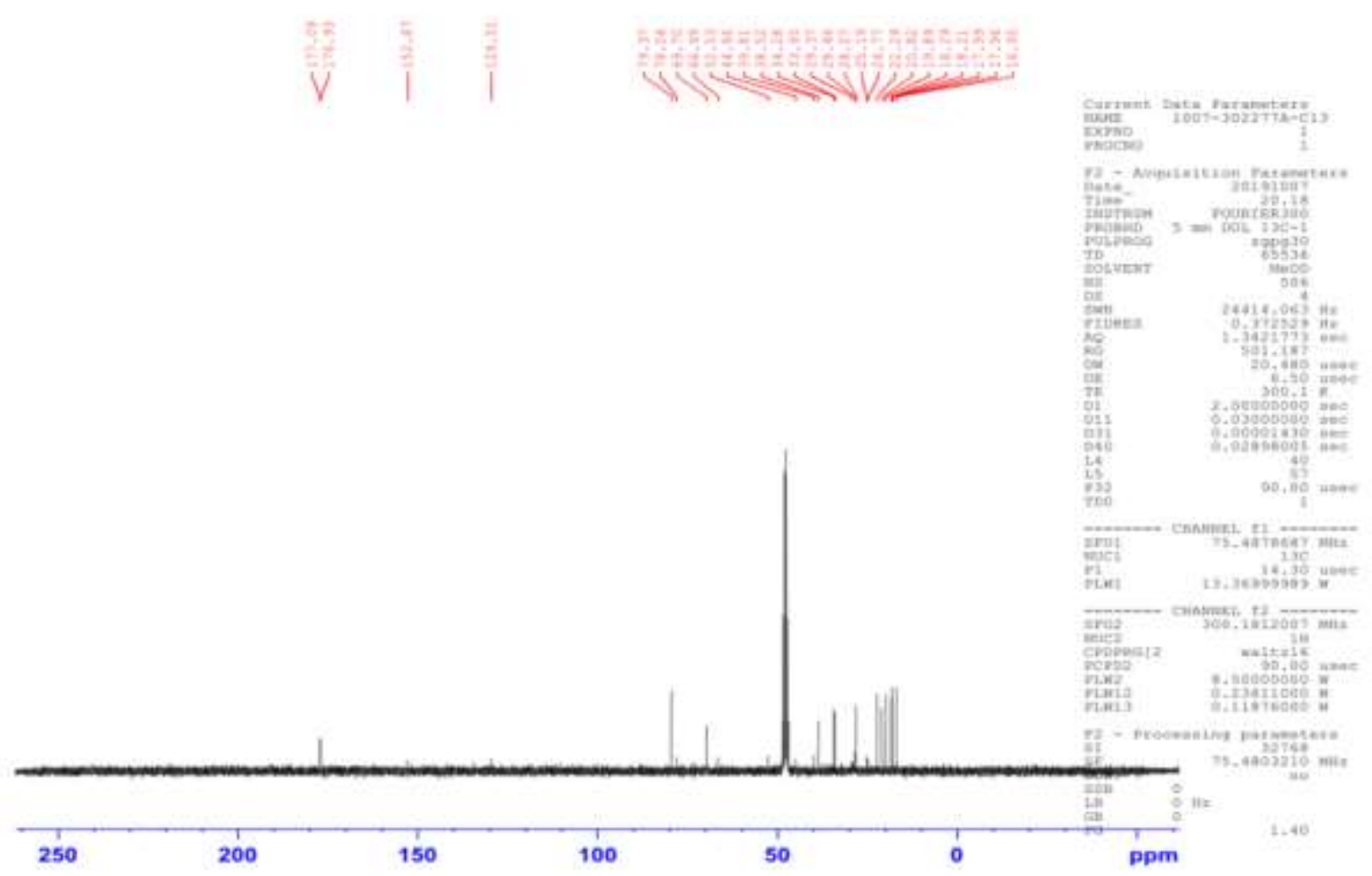


${ }^{13} \mathrm{C}$ Spectrum for compound 12

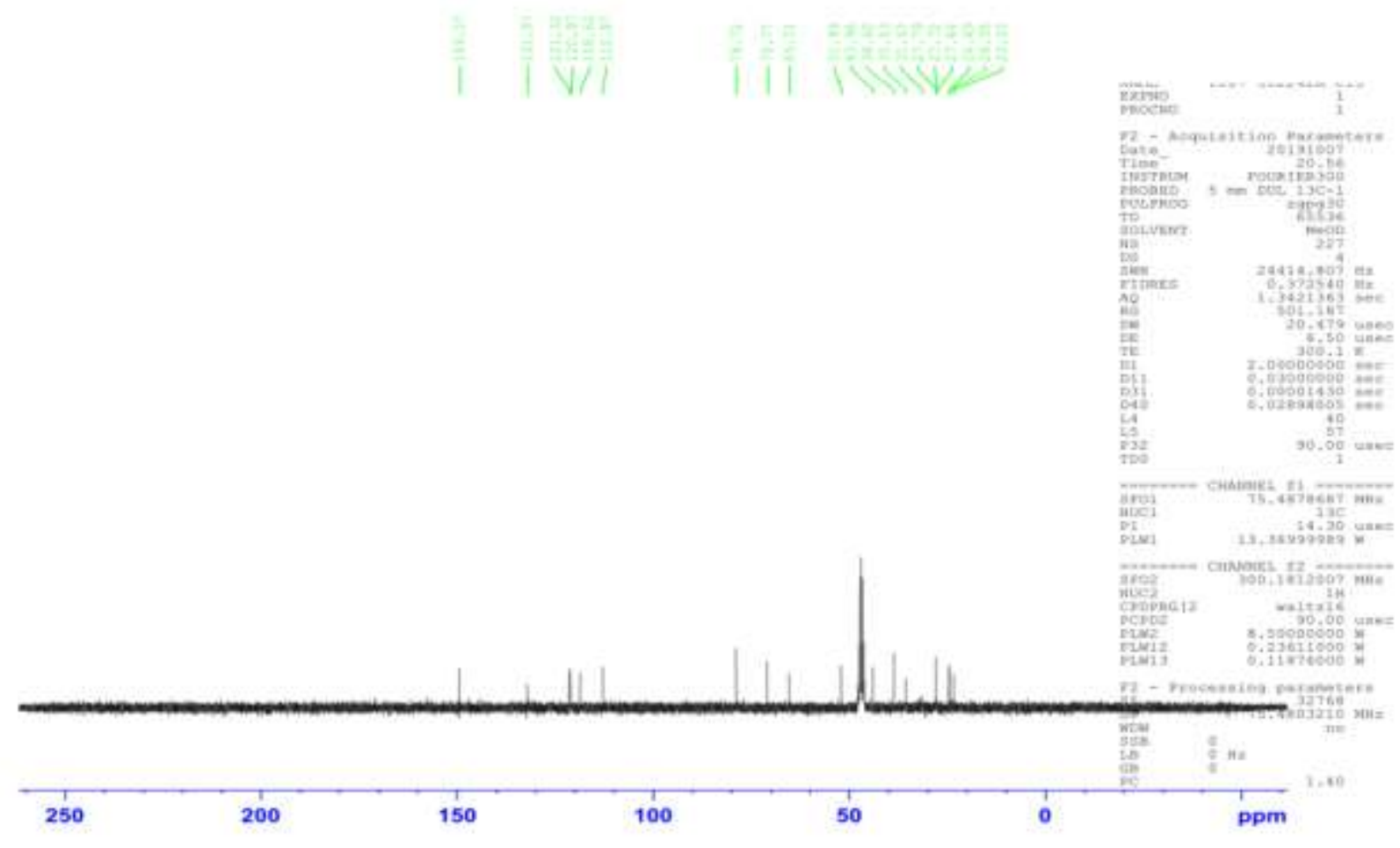


${ }^{13} \mathrm{C}$ Spectrum for compound 13

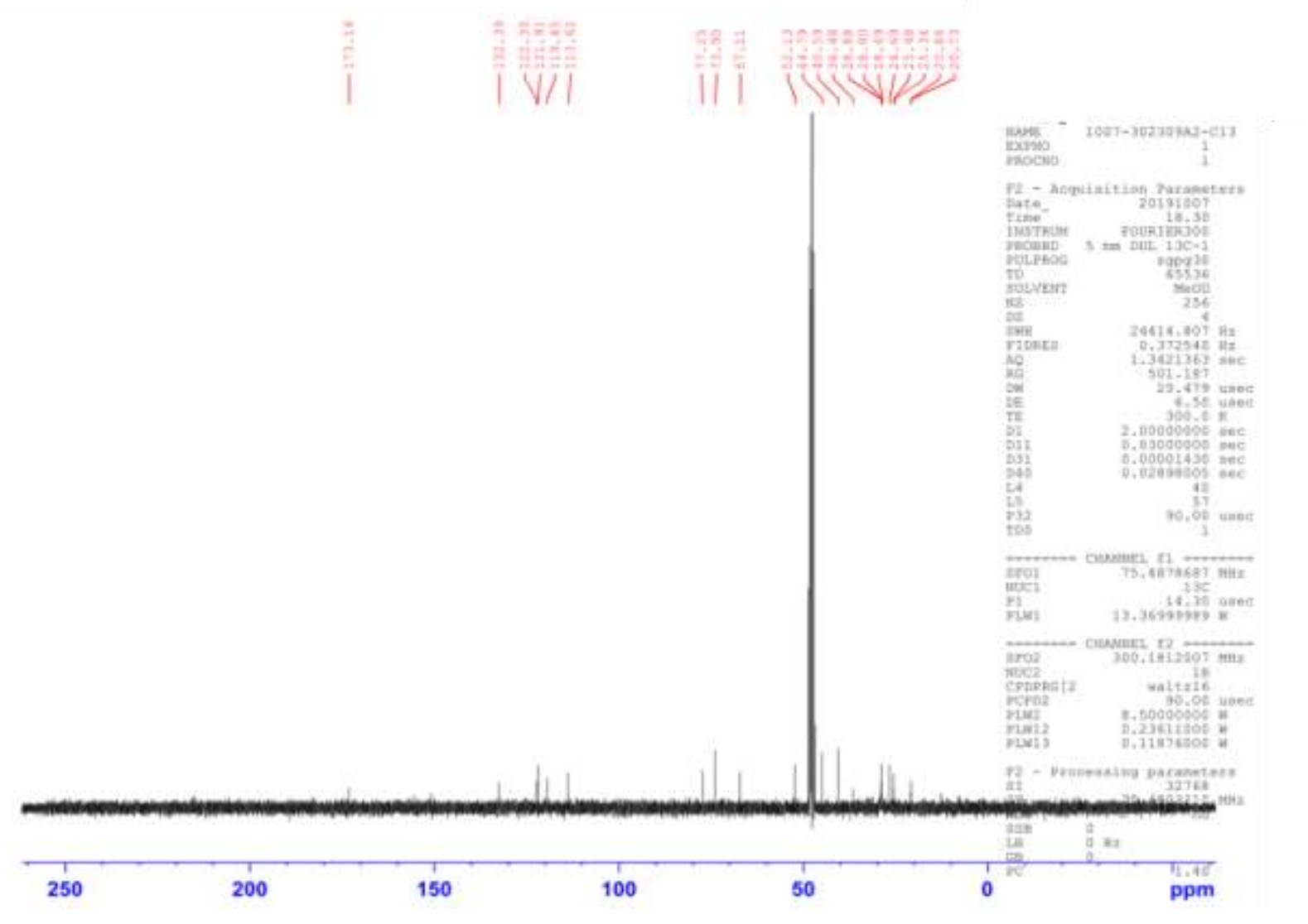


${ }^{13} \mathrm{C}$ Spectrum for compound 14

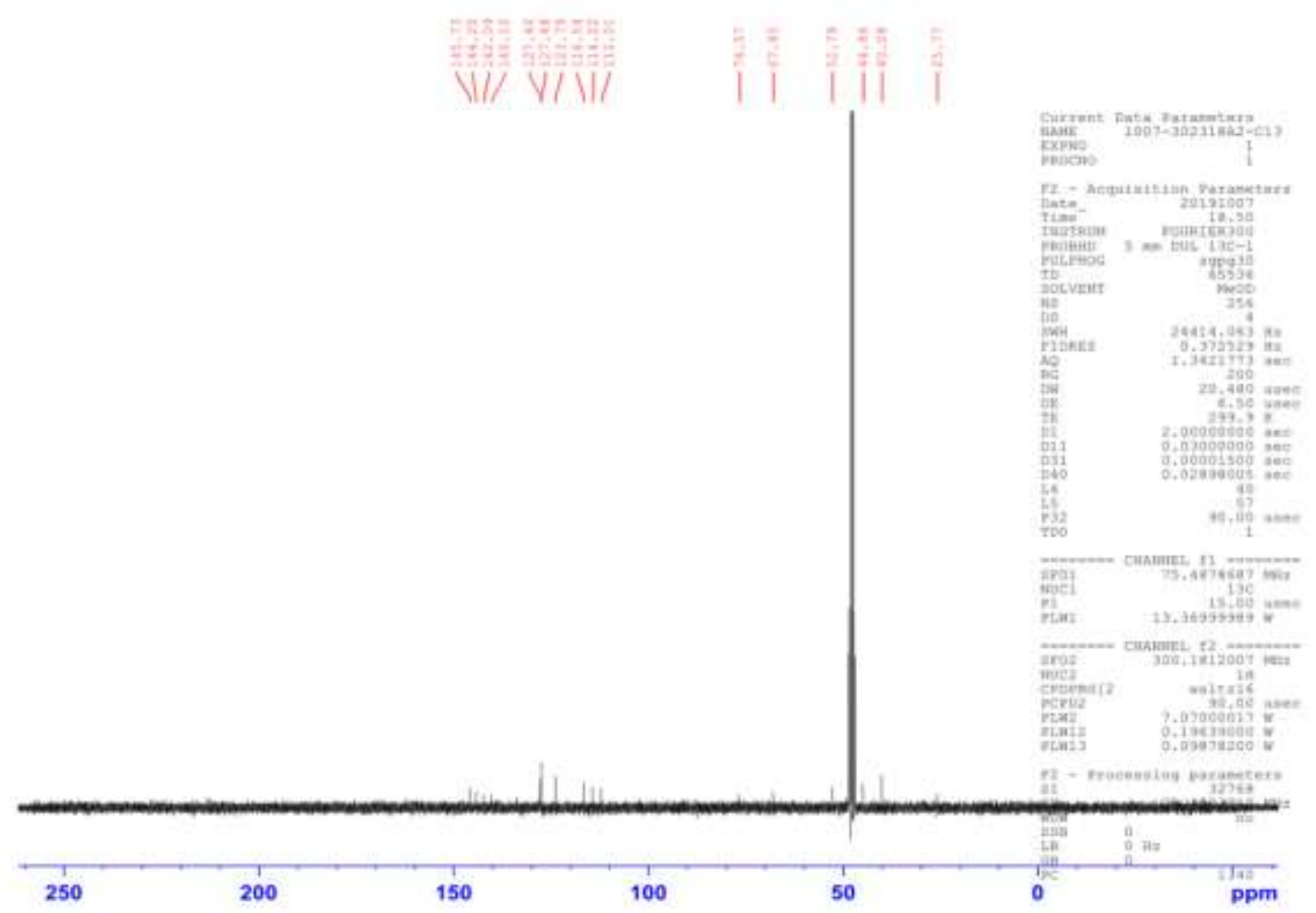


${ }^{13} \mathrm{C}$ Spectrum for compound 15

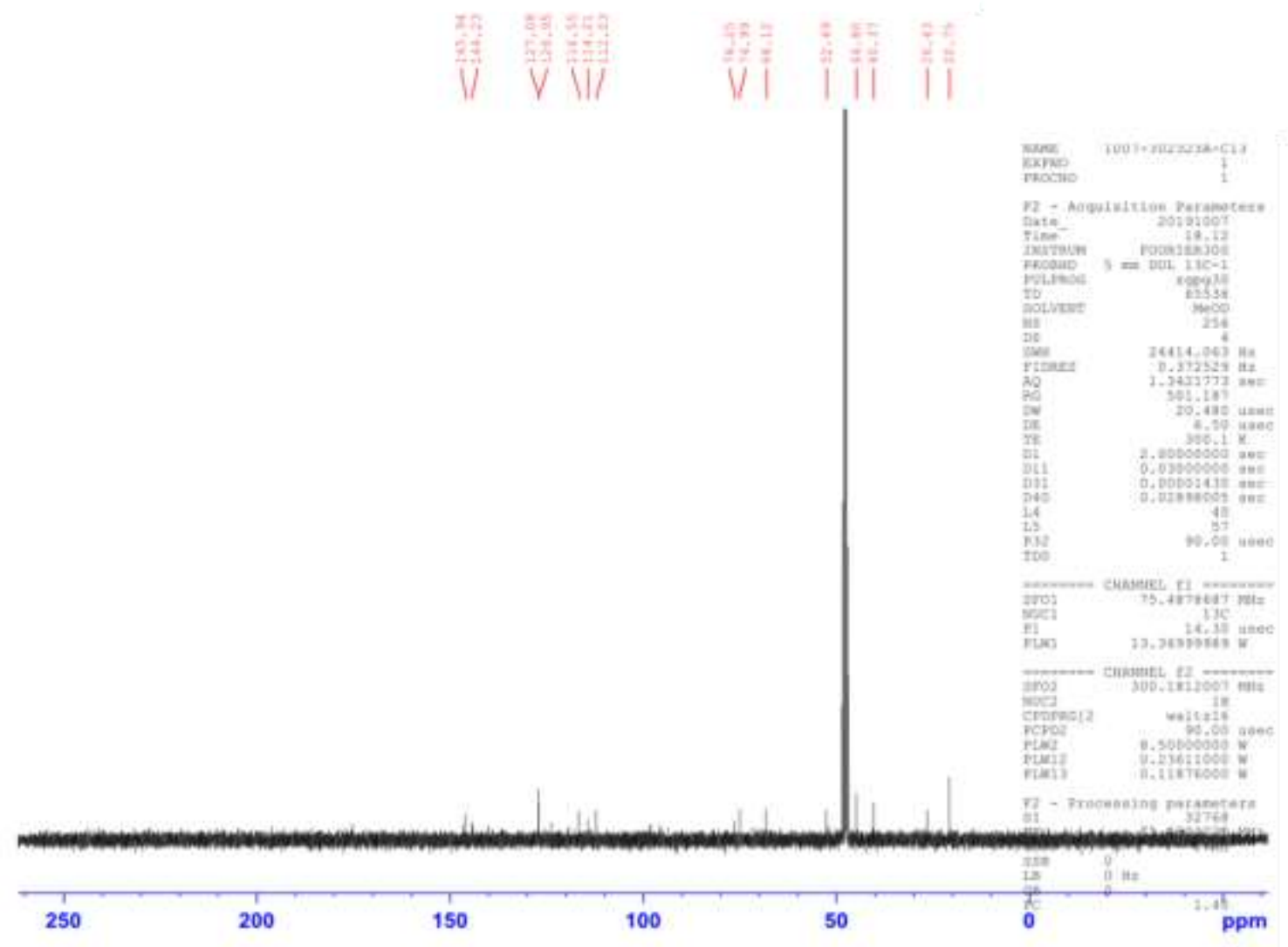




\section{HRMS Spectrum for compound 8}

\section{Elemental Composition Report}

Page 1

Single Mass Analysis

Tolerance $=200.0$ PPM 7 DBE $\min =-10.0, \max =100.0$

Elemant prodiction: Oft
Number of isotope peaks used for i FIT - 3

Monoisolooc Mass. Even Election ions

1309 formulajej evaluated with 326 resuls within limits cup 1020 closest resunts for esch masa)

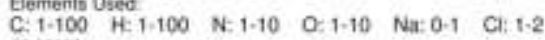

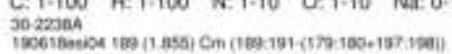

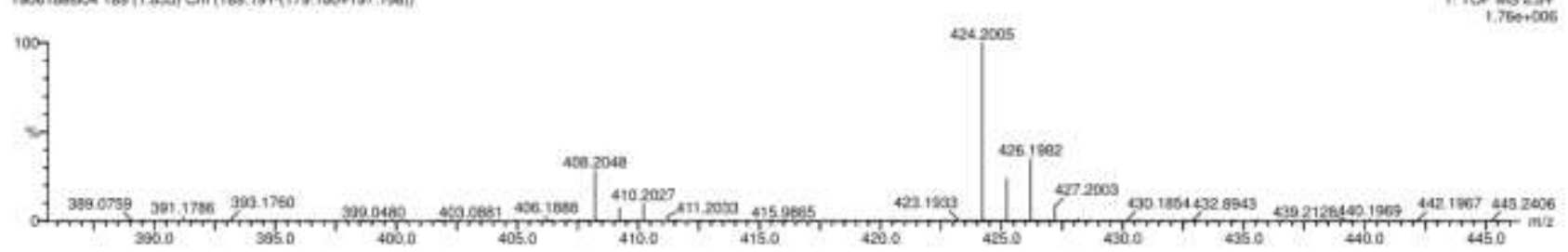

Hinisa: $\quad 5.0 \quad 200.0 \quad \frac{10,0}{100.0}$

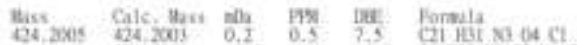

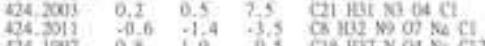

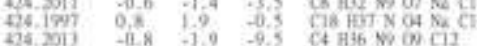

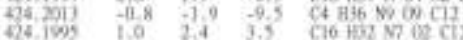

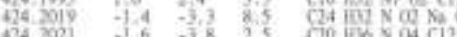

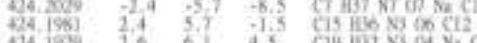

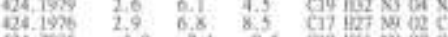

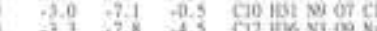

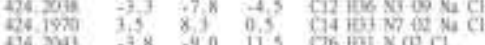

4.4.

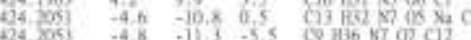

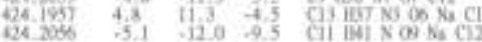




\section{HRMS Spectrum for compound 9}

\section{Elemental Composition Report}

Page 1

Single Mass Analysis

Tolerance $=200.0 \mathrm{PPM}$ / DEE: $\min =-10.0, \max =100.0$

Element prediction: Off

Number of isolope peaks used for i-FIT $=3$

Mocoisoospic Mass. Even Electron lons

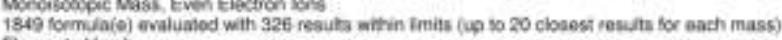

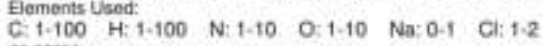

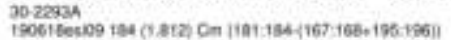

1: tor MS Es,

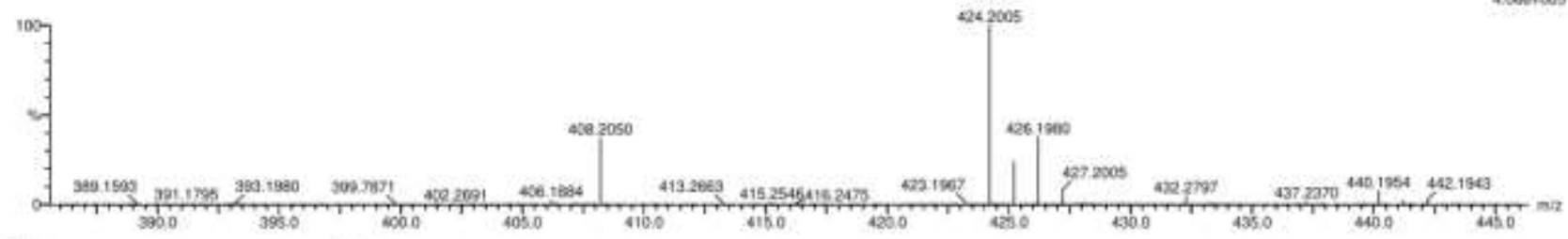

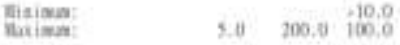

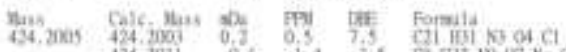

4.2.

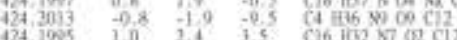

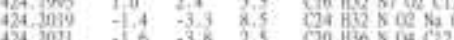

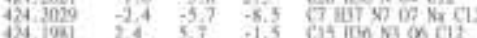

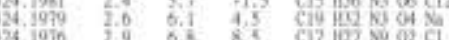

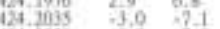

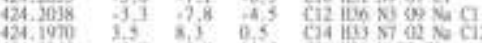

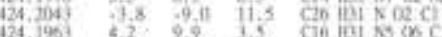

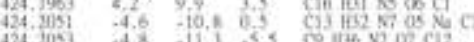

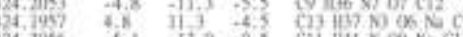


HRMS Spectrum for compound 10

Elemental Composition Report

Page 1

Single Mass Analysis

Tolerance $=200.0$ PPM $/$ DEE: $\min =-10.0, \max =100.0$

Element prediction: On:

Number of igctope peaks used for iFIT = 3

Monoisotopic Mass. Even Election ione

1849 formula(e) evaluated with 328 sesules within limts. (up to 20 ciosest resuls for esch mass

C: $1-100 \quad H: 1-100 \quad N: 1-10 \quad 0: 1-10 \quad \mathrm{Na}: 0-1 \quad$ C: $1-2$

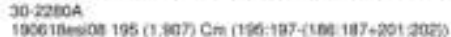

1. TOF us ES.

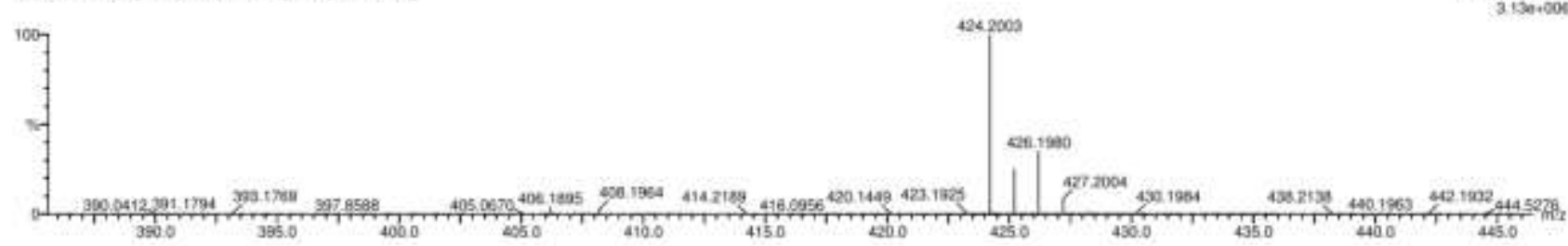

Mininat:
Higing

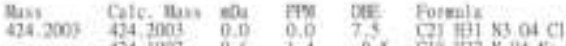

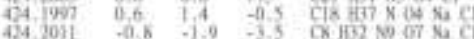

4240550.8 is 35 c16 $392 \mathrm{Nr}^{\circ} \mathrm{Cl}$

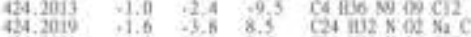

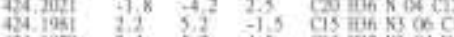

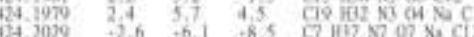

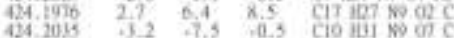

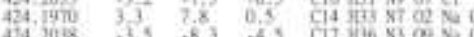

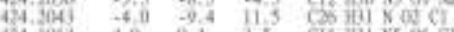

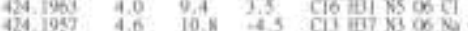

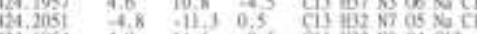

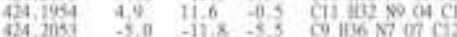


HRMS Spectrum for compound 11

\section{Elemental Composition Report}

Single Mass Analysis

Tolerance $=200.0$ PPM | DBE: $\min =-10.0, \max =100.0$

Element prediction: OIf

Number of iootope peaks used tor ifFT - 3

Monoisoiope Mass. Even Election lons

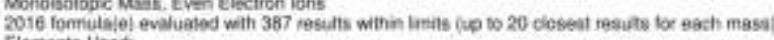

C. $1-100 \quad$ H:1-100 N:1-10 O: 1-10 Na:0-1 Cl: 1-2

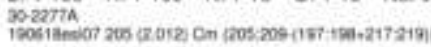

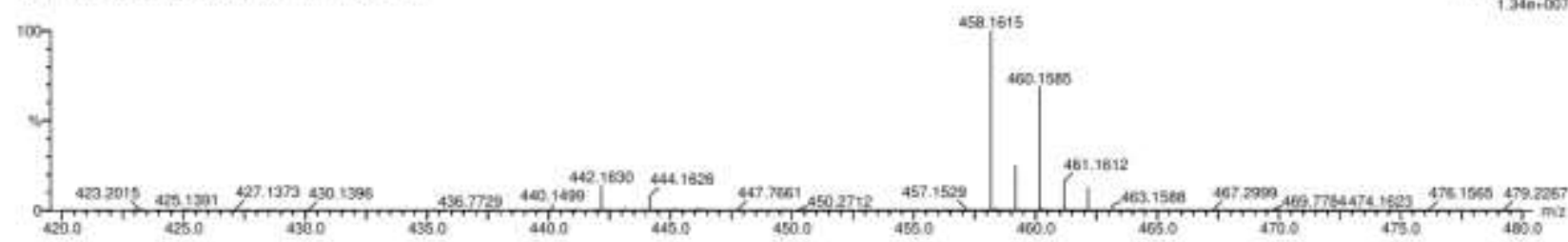

Miniesan: $\quad 5.0 \quad 200.0 \quad 10.0$

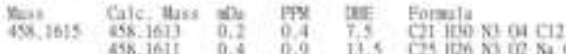

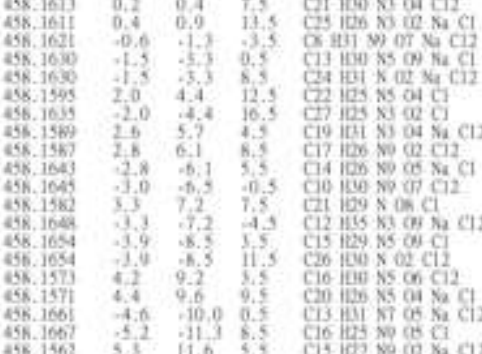


HRMS Spectrum for compound 12

\section{Elemental Composition Report}

Page 1

Single Mass Analysis

Tolerance $=200.0$ PPM ? DeE: $\min =-10.0, \max =100.0$

Number of isotopo poaks used tor i FIT $=3$

Monoisonopic Mass, Even Eloctron lores

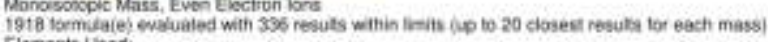

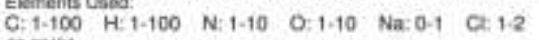

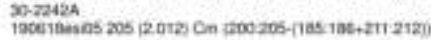

t. TOF Nos ES+

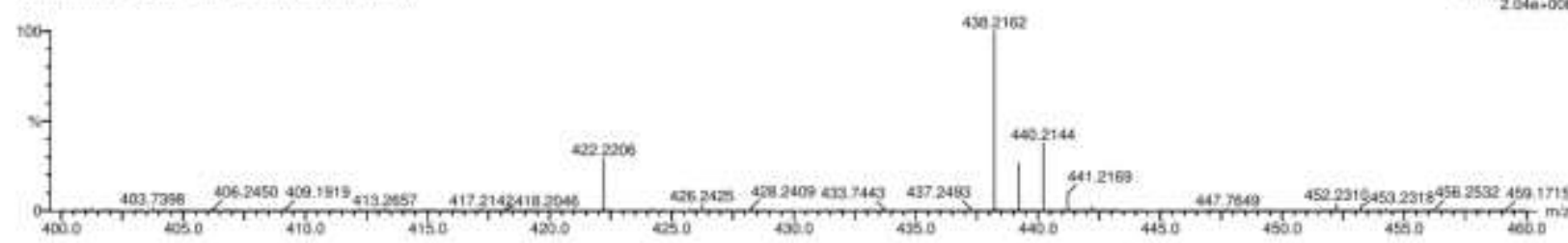

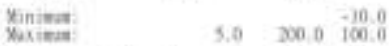

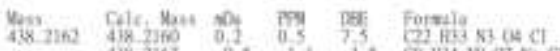

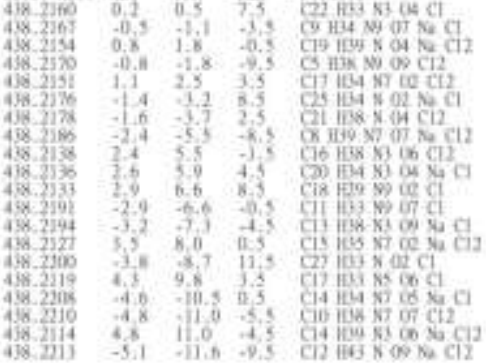


HRMS Spectrum for compound 13

\section{Elemental Composition Report}

\section{Single Mass Analysis}

Tolerance $=200.0$ PPM / DBE: $\min =-10.0, \max =100.0$

Element prediction: Off

Number of isotope peaks used for $\mathrm{i}-\mathrm{FIT}=3$

Monoisotopic Mass, Even Electron lons

1986 formula(e) evaluated with 345 results within limits (up to 20 closest results for each mass)

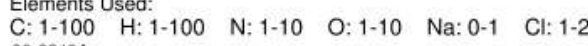

30-2243A

90618esio6 204 (2.003) Cm (204:205-(190:191+215:216))

1: TOF MS ES+

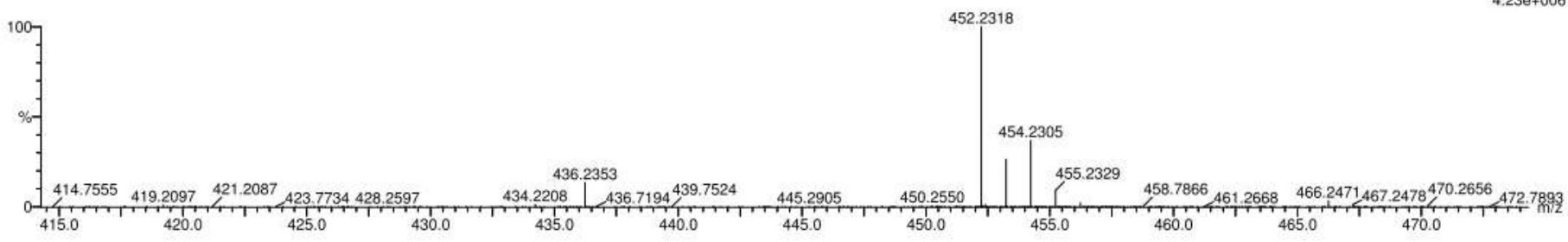

$\begin{array}{llll}\text { Minimum: } & 5.0 \quad 200.0 & -100.0 \\ \text { Maximum: } & 100.0\end{array}$

$\begin{array}{lllllll}\text { Mass } & \text { Calc. Mass } & \mathrm{mDa} & \text { PPNI } & \text { DBE } & \text { Formula } \\ 452.2318 & 452.2316 & 0.2 & 0.4 & 7.5 & \text { C23 H35 N3 04 Cl }\end{array}$

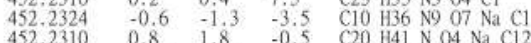

$\begin{array}{llllll}452.2310 & 0.8 & 1.8 & -0.5 & \mathrm{C} 20 \mathrm{H} 41 \mathrm{~N} 04 \mathrm{Na} \mathrm{Cl} \\ 452.2326 & -0.8 & -1.8 & -9.5 & \mathrm{C} 6 \mathrm{H} 40 \mathrm{NO} 09 \mathrm{Cl} 2\end{array}$

$\begin{array}{llllll}452.2308 & 1.0 & 2.2 & 3.5 & \mathrm{C} 18 \text { H36 N7 } 02 \mathrm{Cl} 2\end{array}$

$\begin{array}{llllll}452.2332 & -1.4 & -3.1 & 8.5 & \mathrm{C} 26 \mathrm{H} 36 & \mathrm{~N} \mathrm{O2} \mathrm{Na} \mathrm{Cl} \\ 452.2334 & -1.6 & -3.5 & 2.5 & \mathrm{C} 22 \mathrm{H} 40 \mathrm{~N} \mathrm{O4} \mathrm{C12}\end{array}$

$\begin{array}{llllll}452.234 & -1.6 & -3.5 & 2.5 & \mathrm{C} 22 \\ 452.2342 & -2.4 & -5.3 & -8.5 & \mathrm{C} 9 \mathrm{H} 41 \mathrm{~N} 707 \mathrm{Na} \mathrm{C12}\end{array}$

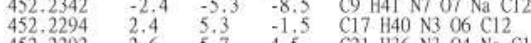

$\begin{array}{llllll}452.2292 & 2.6 & 5.7 & 4.5 & \mathrm{C} 21 \mathrm{H} 36 \mathrm{N3} \mathrm{O4} \mathrm{Na} \mathrm{Cl} \\ 452.2289 & 2.9 & 6.4 & 8.5 & \mathrm{C} 19 \mathrm{H} 31 \mathrm{N9} O 2 \mathrm{Cl}\end{array}$

$\begin{array}{llllll}452.2348 & -3.0 & -6.6 & -0.5 & \text { C12 H35 N9 } 07 \text { Cl }\end{array}$

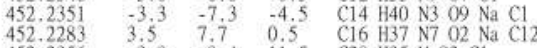

$\begin{array}{llllll}452.2356 & -3.8 & -8.4 & 11.5 & \mathrm{C} 28 & \mathrm{H} 35 \mathrm{~N} \mathrm{O2} \mathrm{Cl}\end{array}$

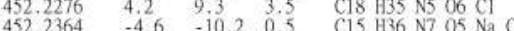

$\begin{array}{llllll}452.2366 & -4.8 & -10.6 & -5.5 & \mathrm{Cl} & \mathrm{H} 40 \mathrm{~N} 707 \mathrm{Cl} 2\end{array}$

$\begin{array}{llllll}452.2270 & 4.8 & 10.6 & -4.5 & \mathrm{C} 15 \mathrm{H} 41 \mathrm{~N} 3 \mathrm{O} 06 \mathrm{Na} \mathrm{Cl} \\ 452.2369 & -5.1 & -11.3 & -9.5 & \mathrm{Cl} 3 \mathrm{H} 45 \mathrm{~N} 09 \mathrm{Na} \mathrm{Cl}\end{array}$ 
HRMS Spectrum for compound 14

\section{Elemental Composition Fieport}

Page 1

Single Mass Analysis

Tolerance $=200.0$ PPM ? DBE: $\min =-10.0, \max =100.0$

Number of isotope poaks used tor i. FIT $=3$

Monoisonopic Mass, Even Electron lans

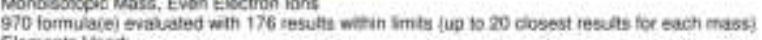

C: $1-100$ H: $1-100 \quad$ N: $1-10$ o: $1-10 \quad$ C: $1-2$

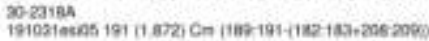

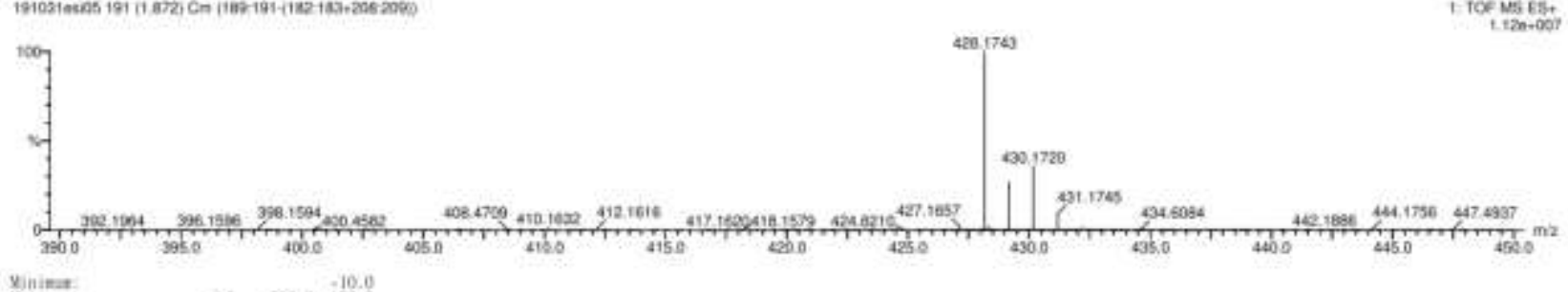

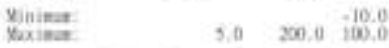

Kas.

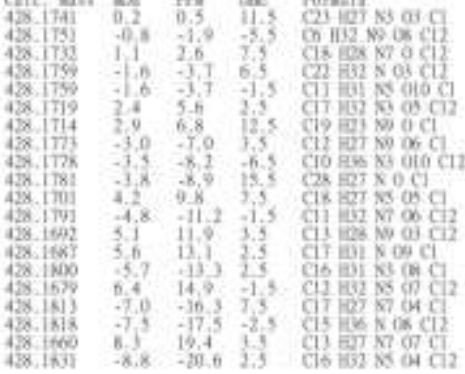


HRMS Spectrum for compound 15

\section{Elemental Composition Report}

Page 1

Single Mass Analysis

Tolerance $=200.0 \mathrm{PPM} / \mathrm{DAE}: \min =-10.0, \max -100.0$

Number of isoliope peaks used tor - FIT - :

Manoisotopic Mass. Evan Electron lons

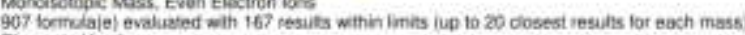

C: $1-100 \quad H=1-100 \quad N: 1-10 \quad 0: 1-10 \quad$ Cl: $1-2$

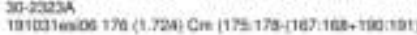

in ToF MSES.

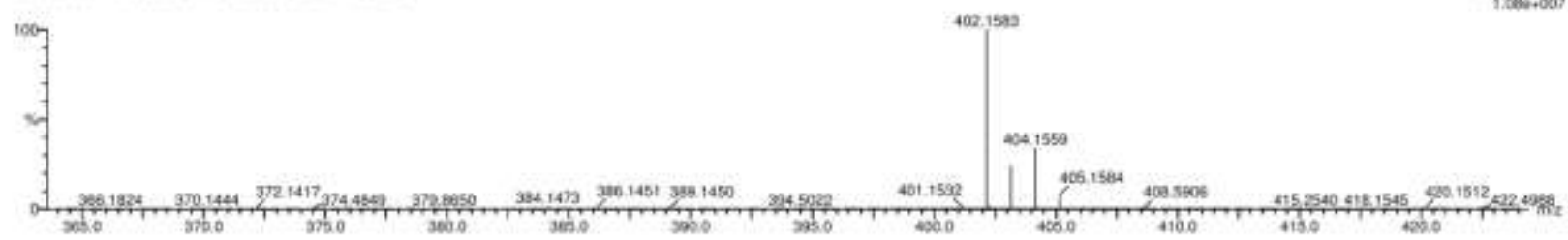

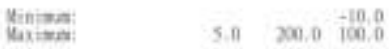

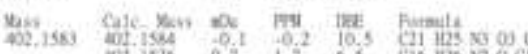

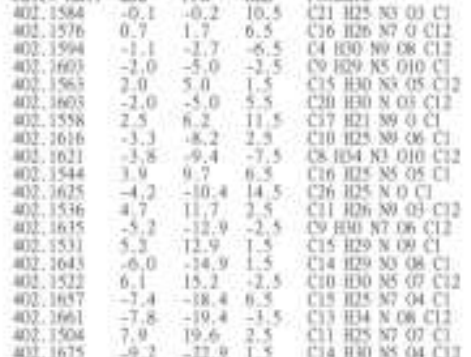

